\title{
Horizon energy and angular momentum from a Hamiltonian perspective
}

\author{
Ivan Booth $1, *$ and Stephen Fairhurst ${ }^{1,3}$ \\ 1. Theoretical Physics Institute, Department of Physics, \\ University of Alberta, Edmonton, Alberta, T6G 2J1, Canada \\ 2. Department of Mathematics and Statistics, \\ Memorial University of Newfoundland, St. John's, \\ Newfoundland and Labrador, A1C 5S\%, Canada \\ 3. Department of Physics, University of Wisconsin - Milwaukee, \\ P.O. Box 413, Wisconsin, 53201, U.S.A.
}

\begin{abstract}
Classical black holes and event horizons are highly non-local objects, defined in terms of the causal past of future null infinity. Alternative, (quasi)local definitions are often used in mathematical, quantum, and numerical relativity. These include apparent, trapping, isolated, and dynamical horizons, all of which are closely associated to two-surfaces of zero outward null expansion. In this paper we show that three-surfaces which can be foliated with such two-surfaces are suitable boundaries in both a quasilocal action and a phase space formulation of general relativity. The resulting formalism provides expressions for the quasilocal energy and angular momentum associated with the horizon. The values of the energy and angular momentum are in agreement with those derived from the isolated and dynamical horizon frameworks.
\end{abstract}

*Electronic address: ibooth@math.mun.ca

${ }^{\dagger}$ Electronic address: sfairhur@gravity.phys.uwm.edu 


\section{INTRODUCTION}

The basic ideas of black hole physics are well-known. These foundations, including the singularity, uniqueness, and area increase theorems [1], the laws of black hole mechanics [2], and the identity between those laws and the laws of thermodynamics [3, 4], have been understood for several decades. Perhaps the most remarkable conclusion to be garnered from these results is the realization that black holes, in spite of their apparently exotic origin, are not really so different from other objects studied by physics. Though classically they harbour singularities in spacetime and have interiors that cannot causally effect the rest of the universe, they still have physical properties such as mass, charge, angular momentum, temperature, and entropy. Nevertheless there is at least one fundamental way in which they are very different. With the exception of spacetimes that are known to be globally stationary, the exact location and size of a black hole cannot be determined by local measurements; instead the existence of a black hole event horizon is a property of the causal structure of the spacetime. Further, in traditional black hole physics, physical properties such as mass cannot be determined by local measurements, but instead are only defined with reference to asymptotic structures. This, of course, contrasts quite strongly with most physical objects which can be identified, measured, and understood without reference to the far future and spatial infinity.

Given the non-local nature of event horizons, there has always been an interest in alternative, local, characterizations of black holes. The original alternative black hole signature was the trapped surface [5] and these figure prominently in the definition of apparent horizons 1]. More recently there have been several other (closely related) approaches. These include trapping [6, 7], isolated [8], dynamical [9], and slowly evolving horizons [10]. All of these will be discussed in more detail in Section ஹA but for now the important point to note is that they are all closely related to each other and defined by the convergence/divergence of particular sets of null geodesics. Specifically they all share one common property : the three-surface which they identify as a "horizon" can be foliated with spacelike two-surfaces of vanishing outward null expansion. These alternative notions of horizons are especially suited to studies of dynamic black holes. For an evolving black hole, the location of an event horizon is impossible to determine unless one first understands the causal structure of the entire spacetime. The alternative horizons do not face this problem as their location depends only on local geometry — the calculations involved may be difficult, but at least they are possible.

Thus, alternative horizons are closely associated with the study of black hole evolutions and their interactions with their surroundings. The exchange of energy and angular momentum between a black hole and its environment is one of the most basic physical problems that might be investigated under these circumstances and this question has been addressed under each of the approaches. Various formulae have been proposed for energy and angular momentum associated with a horizon, as well as the flux of these quantities into and out of the hole.

In this paper, we apply Hamiltonian methods to gain further insight into the energy and angular momentum of horizons. Specifically, we examine quasilocal action and Hamiltonian formulations of general relativity on manifolds which have horizons as boundaries. We begin in Section III with a discussion of the action in the spirit of the well-known Brown-York quasilocal action formalism [11, 12] and its non-orthogonal generalizations [13, 14]. We show that any three-surface (regardless of signature) which can be foliated with spacelike two- 
surfaces of vanishing outward null expansion is a suitable boundary in a quasilocal action formulation for general relativity, provided that we fix the intrinsic geometry of those twosurfaces along with the connection on their normal bundles (a quantity closely associated with angular momentum). This boundary information only needs to be fixed in a weak form - up to diffeomorphisms which may "move it around".

If we impose a notion of time on the quasilocal region, then we can perform a Legendre transform of the action and so obtain a Hamiltonian for the spacetime. As in other derivations of a Hamiltonian, the bulk part of this functional is made up of constraints and so vanishes on solutions of the Einstein equations. Thus one can evaluate it on a slice of a solution by examining only the geometry of the boundary. Consequently, we can derive a quasilocal energy expression for these alternative horizons. As usual, the value of the Hamiltonian is sensitive to the precise form of the action. With our boundary conditions, there is a class of acceptable action functionals; the freedom in the action amounts to being allowed to add free functionals of the fixed data to the original form. As a consequence, the formalism fixes the energy only up to free functionals of the fixed data.

In Section [D] we turn from Lagrangian to Hamiltonian arguments and consider the horizons as boundaries from the point of view of phase space, a symplectic structure, and Hamiltonian evolutions of a quasilocal region of spacetime. This investigation helps to further elucidate the allowed freedom in the definition of an energy and also gives us a concrete expression for the angular momentum associated with the horizon. The calculations follow the extended phase space formalism of [15] which is designed for situations where there are fluxes of quantities such as energy and angular momentum through the boundaries. This is in contrast to the standard Hamiltonian formalism which is designed for isolated systems where there can be no flux of these quantities (and so they are conserved).

Applying this analysis, we derive expressions for the energy and the angular momentum associated with the horizon. The energy expression so derived matches the one found using the action/Legendre transform methods - essentially any diffeomorphism invariant functional of the intrinsic boundary two-geometry and the connection on its normal bundle. The new piece of information is the expression for the angular momentum.

Finally, in Section $\nabla$ we compare the Hamiltonian expressions for energy and angular momentum with those arising from the isolated and dynamical horizon calculations. On sections of the horizon which are null, we find that the angular momentum is identical to that obtained in the isolated horizon framework. Additionally, if we restrict our generators of rotation to divergence free vector fields, the associated angular momenta on spacelike sections of the horizon agree with the dynamical horizon values. Next, we turn to the energy. We can greatly restrict the freedom in the energy by requiring that it satisfy a basic property: the value of the energy must transform appropriately with rescalings of the evolution vector field. We find that in this case the natural evolution vector field is the null vector, $\ell$, for which the outward expansion vanishes. This is true even though it is not, in general, tangent to the horizon. Furthermore, the energy associated to a given evolution is then determined up to an overall constant rescaling. This constant can be fixed by appealing to the dynamical horizon framework. With this choice, the energy derived using Hamiltonian methods will satisfy the dynamical horizon flux law. 


\section{LOCAL HORIZONS - A REVIEW}

\section{A. Definitions}

There are several (quasi-)local definitions of a horizon. In this section, we summarize the various definitions, highlighting both their similarities and differences, and emphasizing those features relevant for the action and Hamiltonian calculations that will follow.

Spacelike two-surfaces and their null normal vectors feature prominently in each of the alternative horizon definitions, so we begin by discussing them. For any spacelike two-surface $S$, we can find families of null vectors that are normal to the surfaces. Up to normalization and orientation, there are just two such null directions. If the spacetime is time-oriented we can single out the forward-in-time pointing directions and so, up to rescaling, define two future-pointing null normals $\ell^{a}$ and $n^{a}$. Then, if $\tilde{q}_{a b}$ is the two-metric on $S$, the expansion associated with these vector fields is given by:

$$
\theta_{(\ell)}=\tilde{q}^{a b} \nabla_{a} \ell_{b} \text { and } \theta_{(n)}=\tilde{q}^{a b} \nabla_{a} n_{b}
$$

These are expansions in the sense that if one Lie-drags $S$ in the direction $\ell^{a}$, and $\widetilde{\boldsymbol{\epsilon}}$ is the area two-form on $S$, then

$$
\mathcal{L}_{\ell} \widetilde{\boldsymbol{\epsilon}}=\widetilde{\boldsymbol{\epsilon}} \theta_{(\ell)}
$$

Thus, $\theta_{(\ell)}$ describes how the area-form on $S$ expands/contracts along $\ell$. Similar arguments apply to $\theta_{(n)}$.

The two-surface $S$ is said to be trapped if it is closed and the expansions of both $\ell$ and $n$ are negative at every point on $S$. A marginally trapped surface is a slight generalization of a trapped surface; in this case, the expansions of the null vectors are required to be less than or equal to zero. Trapped and marginally trapped surfaces can be contrasted to surfaces in flat spacetime where one would expect the outward expansion of convex surfaces to be positive everywhere, and the inward expansion negative. Intuitively, the negative expansion of both null normals can be attributed to strong gravitational fields in the interior of $S$. This picture is strengthened by the singularity theorems which state that (provided energy conditions are satisfied), any spacetime containing a trapped surface must also contain a singularity [1, 16]. Furthermore, it can be shown that any trapped surface in an asymptotically flat spacetime must be contained within an event horizon. Even in the absence of an appropriate asymptotic structure, a trapped surface may always be extended into a spacelike three-surface that hides its inside from its outside [17]. Thus, it seems natural that the various notions of a horizon discussed below all make use of trapped or marginally trapped surfaces.

We begin with a brief discussion of apparent horizons [1]. Let $\Sigma$ be an asymptotically flat Cauchy surface. From an intuitive point of view, such a $\Sigma$ is an "instant" in an asymptotically flat spacetime. Now, a point $p \in \Sigma$ is said to be trapped if it lies on a trapped surface that in turn is contained entirely in $\Sigma$; the total trapped region $\mathcal{T}$ is the union of all the trapped points in $\Sigma$. An apparent horizon is then defined as the boundary $\mathcal{A}$ of the total trapped region $\mathcal{T}$. With certain assumptions about smoothness [18], it can be shown, as one would expect, that $\theta_{(\ell)}=0$ on $\mathcal{A}$. Further, it is true that $\mathcal{T}$ is itself contained within an event horizon. In the case of stationary black hole solutions, such as Schwarzschild, $\mathcal{T}$ completely fills the region of $\Sigma$ inside the black hole and the apparent horizon coincides with the event horizon. 
While it is certainly possible to identify an apparent horizon without reference to the far future, it is not a trivial task to first locate all the trapped surfaces, find their union and then obtain the boundary of that region. Further, one requires knowledge of the entire "instant" before one can locate the apparent horizon. Finally, $\mathcal{A}$ won't always satisfy the smoothness assumptions mentioned in the previous paragraph (see [19] for a good discussion). All of these factors can make working with $\mathcal{A}$ inconvenient (at the very least).

More recent quasilocal horizon definitions attempt to extract the idea of apparent horizons as the boundaries of regions of especially strong gravitational fields, while dropping some of their more unwieldy aspects. Specifically they circumvent a consideration of entire trapped regions and focus directly on two-surfaces $S$ of vanishing outward null expansion $\theta_{(\ell)}=0$. This has the advantage of making the definitions quasilocal. Furthermore, as is the case for apparent horizons, in asymptotically flat spacetimes such surfaces $S$ are always contained within event horizons. In common usage, for example in numerical relativity [20], the term "apparent horizon" is frequently used to refer to the outermost two-surface for which $\theta_{(\ell)}=0$ as it is simply not practical to find the boundary of the total trapped region. However, one should keep in mind that while in some circumstances the definitions are equivalent, these surfaces do not always coincide with $\mathcal{A}$.

Notions such as trapping, isolated, and dynamical horizons seek to formalize these vernacular ideas of an apparent horizon and at the same time remove their somewhat problematic dependence on $\Sigma$ slicing. ${ }^{1}$ Rather than considering two-dimensional surfaces with vanishing outward expansion in some three-surface $\Sigma$, they instead define three-dimensional horizons within the full four-dimensional spacetime. These horizons are required to be foliated by closed spacelike two-surfaces $S_{v}$ on which $\theta_{(\ell)}=0$. Thus, while slicings of the spacetime may be found that are compatible with these $\theta_{(\ell)}=0$ surfaces (in which case they will often be apparent horizons), the horizon definitions are independent of any such foliation of the spacetime. $^{2}$

We will begin by considering isolated horizons and then move on to the very closely related trapping and dynamical horizons. For detailed discussions of the merits of each approach the reader is referred to the literature. Here we will briefly review their relevant properties and demonstrate that each falls within the scope of the upcoming calculations.

Isolated horizons [8] are intended to characterize black holes that are not interacting with their surroundings, even though those surroundings may themselves be dynamic. An isolated horizon is a null three-surface whose null normal has zero expansion. Every foliation of an isolated horizon will have the same outward null normal, whence the foliation into twosurfaces is not important. Making use of a mild energy condition, it is straightforward to demonstrate that isolated horizons don't change in either shape or size and no energy flows across them. With an additional restriction on the permitted class of null normals $\ell^{a}$, it can be shown that isolated horizons obey the zeroth law of black hole mechanics, namely that the surface gravity is constant over the horizon. Furthermore, a phase space version of the first law has been shown to hold. Finally, loop quantum gravity calculations have demonstrated that they have an entropy proportional to their surface area [22].

\footnotetext{
${ }^{1}$ For example, it is well-known that even Schwarzschild spacetime may be foliated so that there are no apparent horizons in any slice 21].

2 Note however that the slicing dependence discussed in the last footnote is then replaced by a non-rigidity of the horizons. In general the two-surfaces making up these horizons may be smoothly deformed while keeping their $\theta_{(\ell)}=0$ property. See 25] for a recent discussion of this non-uniqueness.
} 
Trapping horizons [6] are defined as three-surfaces (of indeterminate signature) that can be foliated with $\theta_{(\ell)}=0$ two-surfaces $S$. By themselves these conditions are not enough to distinguish between outer and inner black hole horizons or for that matter between black holes and white holes. Additional conditions are added to define future outer trapping horizons (FOTHs). A FOTH is a trapping horizon that also satisfies the property that $\theta_{(n)}<0$ and $\mathcal{L}_{n} \theta_{(\ell)}<0$. Thus, ingoing null geodesics are converging, while outgoing null geodesics converge inside the horizon, diverge outside and have zero expansion on the horizon. Given a three-surface $\Sigma$ that intersects a FOTH along a foliating two-surface $S$, these conditions are intended to ensure that the region inside of $S$ is trapped, while the region outside will not be (thus $S$ should be apparent horizon). In a spacetime respecting the null energy condition, the above conditions are sufficient to guarantee that dynamic trapping horizons are spacelike and expanding, and so individually obey a form of the second-law of black hole mechanics. In the absence of (gravitational wave) shearing or matter falling across the horizon, they are null and non-expanding. Finally for energy condition violating matter, horizons can shrink in size, in accord with Hawking radiation.

A slowly evolving horizon [10] is an "almost" isolated FOTH and so defined in the same way but with an extra condition that characterizes "almost". Intuitively, these horizons can be thought of as FOTHs which are only weakly interacting with their environment. In this regime, a truly dynamical version of the first law for black holes has been formulated. This is also the regime of interest for studying the approach to equilibrium after a gravitational collapse or black hole merger.

A dynamical horizon (DH) [9] is also closely related to a FOTH. It is a spacelike threesurface that can be foliated with spacelike two-surfaces for which $\theta_{(n)}<0$ and $\theta_{(\ell)}=0$. Here, the requirement that the surface be spacelike has replaced the $\mathcal{L}_{n} \theta_{(\ell)}<0$ condition of the FOTH. This ensures that the horizon can be identified without the need to take derivatives out of the three-surface. In cases where the horizon is expanding (which are largely the cases of interest) the Einstein equations along with energy conditions ensure that the definitions of dynamical horizons and FOTHs are equivalent. It has been shown that dynamical horizons satisfy a variety of conservation laws that relate the change in horizon properties to fluxes of stress-energy and matter across the horizon. The connection between those laws and the calculations of this paper will be discussed further in Section $\mathrm{V}$

Finally, there has recently been some discussion of non-FOTH/DH extensions of FOTHs/DHs [23, 24, 25]. Referred to as marginally trapped tubes (MTTs), these are three-surfaces that satisfy $\theta_{(\ell)}=0$ and $\theta_{(n)}<0$ but are not not necessarily everywhere null or spacelike. In the terminology of trapping horizons, they are future trapping horizons with the slight generalization that $\mathcal{L}_{n} \theta_{(\ell)}$ may take any value. It has been shown that a single MTT can include not only regions that are isolated and dynamical but also regions with timelike signatures (where $\mathcal{L}_{n} \theta_{(\ell)}<0$ ). Such regions appear to be associated with extreme conditions where new horizons form outside of old ones. Though these so-called timelike membranes probably cannot be viewed as horizons (they are traversible in both directions), they can evolve directly into/from DHs and so need to be kept in mind during the following discussions as possible quasilocal boundaries. That said, MTTs will usually be excluded from consideration in the following as they introduce complications such as non-trivial intersections with any foliation $\Sigma_{t}$ of the spacetime (see [24] for many examples of these complications). 


\section{B. Horizon Geometry}

In the preceeding section, we have discussed many different local horizon formulations. Here, we would like to examine the geometrical properties of the horizons, focussing in particular on those which are common to all the formulations. Initially, we will restrict our attention to two-surfaces $S$ of zero expansion which foliate a three-surface $H$. The intrinsic geometry of the horizon is completely determined by the two-metric $\tilde{q}_{a b}$ and the corresponding area two-form $\widetilde{\boldsymbol{\epsilon}}$ introduced previously. The extrinsic geometry of the surface is partially characterized by the extrinsic curvatures $\left(k_{a b}\right)$ of $S$ along the null directions,

$$
\begin{aligned}
\frac{1}{2} \theta_{(\ell)} \tilde{q}_{a b}+\tilde{q}_{a c} \tilde{q}_{b d} \sigma_{(\ell)}^{c d} & =k_{(\ell) a b}:=\tilde{q}_{a}{ }^{c} \tilde{q}_{b}{ }^{d} \nabla_{c} \ell_{d} \text { and } \\
\frac{1}{2} \theta_{(n)} \tilde{q}_{a b}+\tilde{q}_{a c} \tilde{q}_{b d} \sigma_{(n)}^{c d} & =k_{(n) a b}:=\tilde{q}_{a}{ }^{c} \tilde{q}_{b}{ }^{d} \nabla_{c} n_{d} .
\end{aligned}
$$

Here, $\theta_{(\ell)}$ and $\theta_{(n)}$ are the expansions of the null normals, introduced previously, while $\sigma_{(\ell)}^{a b}$ and $\sigma_{(n)}^{a b}$ are the shears along $\ell$ and $n$ respectively. Since $\ell$ and $n$ are null normals to the surface $S$ they are, by definition, twist free. The expansion of $\ell$ is required to vanish on all the horizons discussed above. The other quantities will generically be non-zero except on an isolated horizon when the shear $\sigma_{(\ell)}^{a b}$ must also vanish as there can be no flux of gravitational energy across the horizon.

The rest of the extrinsic geometry is described by the connection on the normal bundle to $S$,

$$
\omega_{a}=-n_{c} \nabla_{a} \ell^{c}
$$

This quantity is closely associated in many of the formalisms with physical characteristics of the horizons. On an isolated horizon, the surface gravity is obtained by contracting with $\ell$, whence $\kappa_{(\ell)}=\ell^{a} \omega_{a}=-\ell^{a} n_{c} \nabla_{a} \ell^{c}$. Additionally, the two-surface components of $\omega$, namely

$$
\tilde{\omega}_{a}=\tilde{q}_{a}{ }^{b} \omega_{b}=-\tilde{q}_{a}{ }^{b} n_{c} \nabla_{b} \ell^{c},
$$

are often associated with quasilocal angular momentum [8, 9, 11, 26, 27]. Specifically, on an axisymmetric isolated or slowly evolving horizon with a symmetry generated by $\phi^{a}$, the angular momentum is given by

$$
J_{I H}[\phi]=-\frac{1}{8 \pi G} \int_{S} \tilde{\boldsymbol{\epsilon}} \phi^{a} \tilde{\omega}_{a}
$$

The definition of angular momentum on a dynamical horizon is slightly different. Since dynamical horizons are by definition spacelike, we can introduce the timelike unit normal to the horizon $\hat{\tau}^{a}$ and the unit spacelike normal to the leaves of the horizon, $\hat{r}^{a}$. Then, the angular momentum is constructed from these vector fields as

$$
J_{D H}[\phi]=\frac{1}{8 \pi G} \int_{S} \tilde{\boldsymbol{\epsilon}} \phi^{a} \hat{r}^{b} \nabla_{a} \hat{\tau}_{b} .
$$

Then, it is straightforward to integrate the diffeomorphism constraint over the horizon to obtain a flux law for angular momentum [9]. Specifically, given a region of the horizon $\Delta H$ bounded by two surfaces $S_{2}$ and $S_{1}$, the angular momentum satisfies

$$
J_{2}[\phi]-J_{1}[\phi]=\int_{\Delta H}^{(H)} \boldsymbol{\epsilon}\left\{T_{a b} \hat{\tau}^{a} \phi^{b}+\frac{1}{16 \pi G} P_{\hat{\tau}}^{i j} \mathcal{L}_{\phi} q_{i j}\right\} .
$$


In the above ${ }^{(H)} \boldsymbol{\epsilon}$ is the natural volume form on the horizon. The two terms in (2.8) can then be interpreted as the flux of matter and gravitation angular momentum through the horizon.

\section{THE ACTION}

In this section we construct an action formulation for general relativity over a quasilocal region of spacetime that includes a trapping horizon $H$ as one of its boundaries. To do this, we first introduce the spacetime $M$ of interest, and then we specify appropriate boundary conditions on each boundary of the spacetime. Those on the horizon will be specified in terms of the geometrical properties discussed in Section IIB while those on the other boundaries are standard and well-known. Combining these with an action whose bulk term is the usual Einstein-Hilbert term, we show that the first variation of the action vanishes in the required way if the Einstein equations hold in the bulk. Thus, an action formulation exists for spacetimes which have a horizon as a boundary. Finally, by performing a Legendre transform, we derive a corresponding quasilocal Hamiltonian from this action. In the usual way, the form of this functional then provides a definition of the energy associated with the region and its assorted boundaries.

\section{A. The spacetime and its boundaries}

Let $M$ be a finite region of spacetime with metric $g_{a b}$, metric-compatible derivative $\nabla_{a}$, and four-volume form $\epsilon_{a b c d}$ (also written as $\boldsymbol{\epsilon}$ ). We require $M$ to be time orientable and diffeomorphic to $[0,1] \times[0,1] \times S^{2}$, where the four boundaries are:

1. a spacelike past boundary $\Sigma_{1}$ which represents the initial configuration,

2. a spacelike future boundary $\Sigma_{2}$ which represents the final configuration,

3. a timelike outer boundary $B$, and

4. an inner horizon boundary $H$ which is foliated by two-spheres ${ }^{3} S_{v}$ of zero outward null expansion $\left(\theta_{(\ell)}=0\right)$.

The two-dimensional intersections of these boundaries are $\mathcal{B}_{1}=\Sigma_{1} \cap B, \mathcal{B}_{2}=\Sigma_{2} \cap B$, $S_{1}=\Sigma_{1} \cap H$, and $S_{2}=\Sigma_{2} \cap H$. Figure 1 provides a three-dimensional representation of the manifold $M$ and these assorted boundaries.

In describing the precise boundary conditions on each of the surfaces and also in the ensuing calculations, it will often be important to distinguish between four-dimensional quantities and those defined exclusively in the various two- and three-dimensional surfaces. To this end, we introduce some notational conventions. Tensor fields which are elements of the tensor bundles of the full spacetime will be labelled with lower case early alphabet Latin

\footnotetext{
3 Strictly speaking the only property of $S^{2}$ that we will need in the following calculations is that it is closed. That said it can be shown [6] that in most physically interesting situations, the Einstein equations along with energy conditions actually restrict the $S_{v}$ to being topologically $S^{2}$. Thus, for definiteness, we make this assumption.
} 


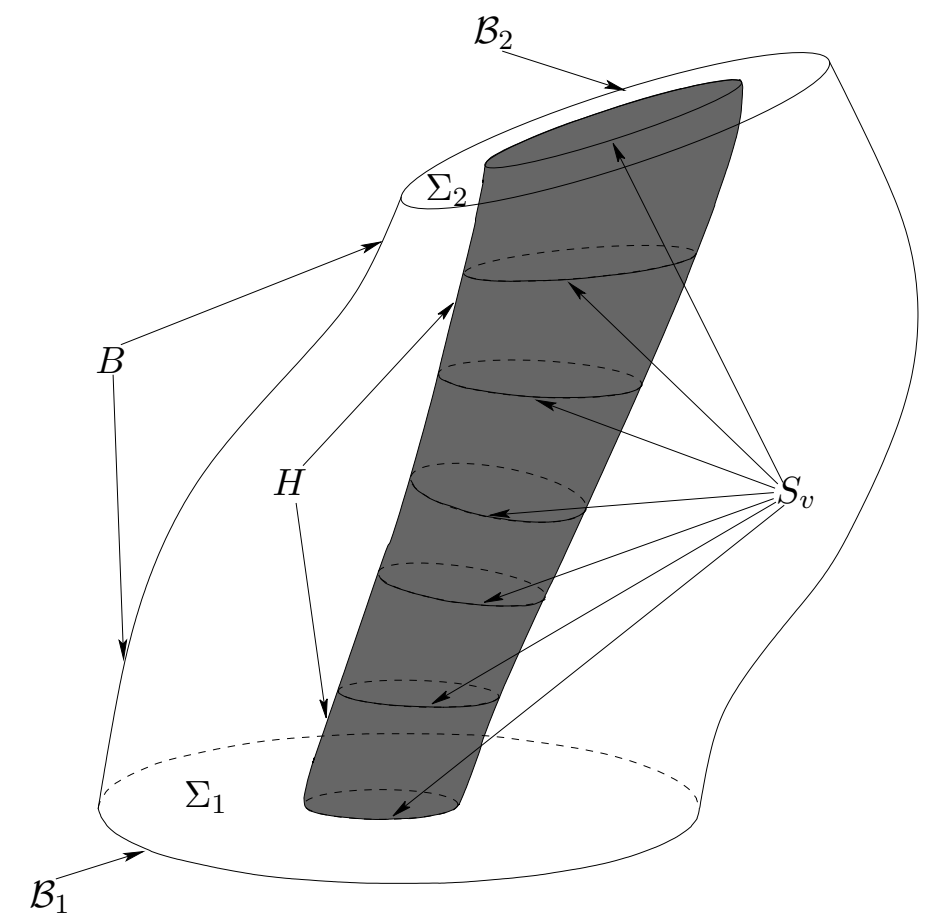

FIG. 1: A three-dimensional representation of $M$ along with its boundaries.

indices $(a, b, c$, etc.). Tensors in the bundles associated with three-dimensional hypersurfaces will be labelled with lower case mid-alphabet Latin indices $(i, j, k$, etc.) and tensors in the bundles associated with two-dimensional surfaces will be labelled with upper case Latin indices $(A, B, C$, etc.). Additionally, we will need to map tensors between the tensor bundles associated with the different manifolds. We will write all pull-back/push-forward operators as $p$ with indices added to indicate which spaces it maps between. Thus, the four-dimensional metric $g_{a b}$ pulled back into a two-dimensional surface would be written $p_{A}{ }^{a} p_{B}^{b} g_{a b}$, while a three-dimensional vector $v^{i}$ would push forward to $p_{i}{ }^{a} v^{i}$ in the full spacetime. Of course, as indicated by the indices, only fully covariant tensors may be pulled back and only fully contravariant tensors may be pushed forward in a metric independent way. If we wish to project tensors of a different rank we must make use of the metric, rendering the results metric dependent.

With the preliminaries out of the way, we now describe in more detail the various boundaries of $M$. Figure 2 provides a two-dimensional representation of the spacetime $M$ and its boundaries. Surface normals and other important vector fields are also shown.

Initial and final boundaries : $\Sigma_{1}, \Sigma_{2}$

Denote the future directed unit normal vectors to the spacelike boundaries $\Sigma_{1,2}$ by $\hat{u}^{a}$. Then, the three-metric on the surfaces $\Sigma$ is given by $h_{i j}$ which satisfies $h^{a b}:=p_{i}{ }^{a} p_{j}{ }^{b} h^{i j}=$ $g^{a b}+\hat{u}^{a} \hat{u}^{b}$. The metric compatible derivative operator is $D_{i}$. We obtain the three-volume 


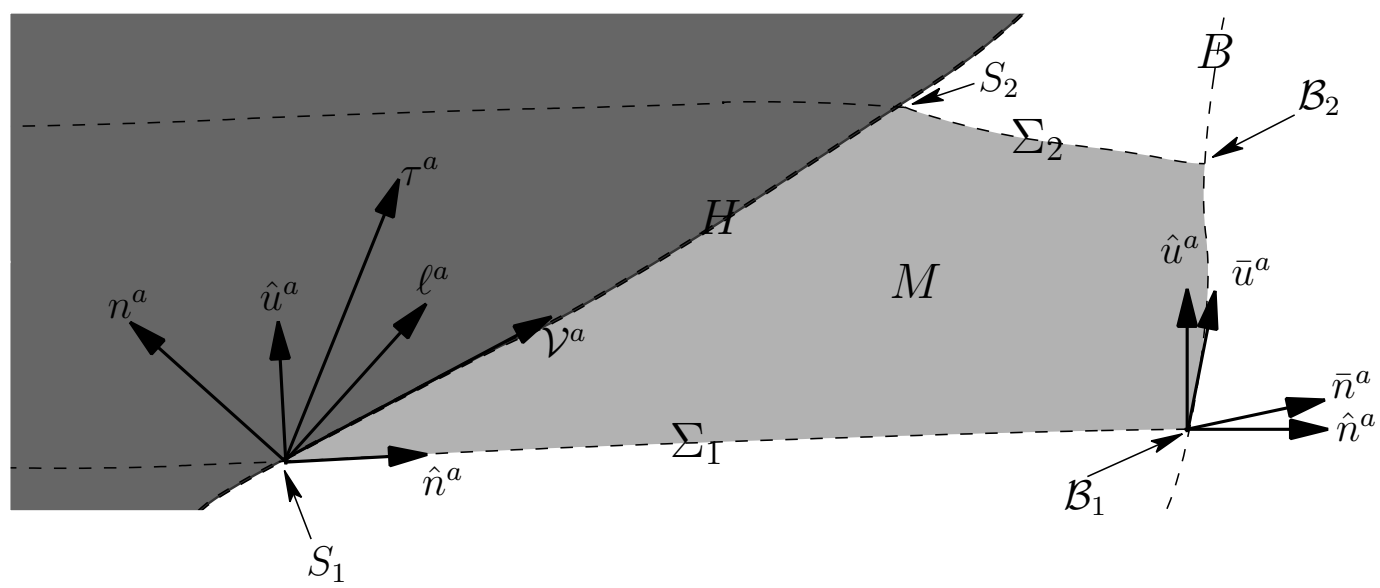

FIG. 2: A two-dimensional representation of the spacetime containing $M$ (the lightly shaded region), showing the past and future three-boundaries $\Sigma_{1}$ and $\Sigma_{2}$ along with the inner and outer three-boundaries $H$ and $B$. The intersections of these surfaces $S_{1,2}$ and $\mathcal{B}_{1,2}$ are also shown along with the orientations of the assorted normal vector fields for the case where $H$ is spacelike and expanding. The darkly shaded region is the interior of $H$ - the "hole". Each point of this schematic is a two-sphere in the real $M$.

form by contracting the four-volume form with the normal $\hat{u}^{a} .{ }^{4}$ Thus, ${ }^{(\Sigma)} \epsilon_{a b c}=\epsilon_{a b c d} \hat{u}^{d}$ from which it follows that $\boldsymbol{\epsilon}=\hat{\mathbf{u}} \wedge^{(\Sigma)} \boldsymbol{\epsilon}$. Finally, the extrinsic curvature of $\Sigma$ in $M$ is given by $K_{(\hat{u})}^{a b}=h^{a c} h^{b d} \nabla_{c} \hat{u}_{d}{ }^{5}$

Outer boundaries : $B, \mathcal{B}_{1}, \mathcal{B}_{2}$

In keeping with the standard quasilocal action analyses, we take the outer boundary $B$ to be timelike with $\bar{n}^{a}$ as its outward pointing spacelike unit normal. Then, the threemetric on $B$ is $\gamma_{i j}$, where $\gamma^{a b}=p_{i}{ }^{a} p_{j}{ }^{b} \gamma^{i j}=g^{a b}-\bar{n}^{a} \bar{n}^{b}$ and it defines the metric compatible derivative $\Delta_{i}$. The extrinsic curvature $K_{(\bar{n})}^{a b}=\gamma^{a c} \gamma^{b d} \nabla_{c} \bar{n}_{d}$ and the volume form is taken to be ${ }^{(B)} \boldsymbol{\epsilon}_{a b c}=-\epsilon_{a b c d} \bar{n}^{d}$, so that $\boldsymbol{\epsilon}=\overline{\mathbf{n}} \wedge^{(B)} \boldsymbol{\epsilon}^{6}$

The outer boundary $B$ intersects $\Sigma_{1}$ and $\Sigma_{2}$ on the spacelike two-surfaces $\mathcal{B}_{1}$ and $\mathcal{B}_{2}$. We do not require that this intersection be orthogonal (i.e. there is no requirement that $\hat{u} \cdot \bar{n}=0$ ). Thus, we introduce two additional normals: $\hat{n}^{a}$, the outward unit spacelike normal

${ }^{4}$ The volume form is induced on every submanifold by Stokes theorem. It can be found by contracting $\epsilon_{a b c d}$ with the outward normal on the last index.

${ }^{5}$ For those familiar with the work of Brown and York [1], note that they define extrinsic curvature with the opposite sign.

${ }^{6}$ Note that this is the opposite of the volume form induced by Stokes theorem. Consequently we will introduce a minus sign when integrating an exact derivative out to the boundary $B$. 
to $\mathcal{B}_{1,2}$ in $\Sigma_{1,2}$, and $\bar{u}^{a}$, the future directed timelike unit normal to $\mathcal{B}_{1,2}$ in $B$ (as illustrated in figure 2). Defining $\eta=-\hat{u} \cdot \bar{n}$, the unit normals are related by

$$
\bar{u}^{a}=\sqrt{1+\eta^{2}} \hat{u}^{a}+\eta \hat{n}^{a} \text { and } \bar{n}^{a}=\eta \hat{u}^{a}+\sqrt{1+\eta^{2}} \hat{n}^{a} .
$$

Clearly, when $\eta=0, \bar{u}^{a}=\hat{u}^{a}$ and $\bar{n}^{a}=\hat{n}^{a}$ and so the $\Sigma / B$ intersection is orthogonal.

The two-metric on $\mathcal{B}_{1,2}$ is denoted $\bar{\gamma}_{A B}$ and its inverse pushes forward to $\bar{\gamma}^{a b}=h^{a b}-\hat{n}^{a} \hat{n}^{b}=$ $\gamma^{a b}+\bar{u}^{a} \bar{u}^{b}$. The derivative operator compatible with this metric is $d_{A}$. The volume form $\overline{\boldsymbol{\epsilon}}$ on $\mathcal{B}_{1,2}$, is given by $\boldsymbol{\epsilon}=\overline{\mathbf{u}} \wedge \overline{\mathbf{n}} \wedge \overline{\boldsymbol{\epsilon}}=\hat{\mathbf{u}} \wedge \hat{\mathbf{n}} \wedge \overline{\boldsymbol{\epsilon}}$.

Inner boundaries: $H, S_{1}, S_{2}$

A discussion of the inner boundary $H$ is more involved than that of the outer boundary. Since this boundary is required to be a trapping horizon:

1. $H$ has additional structure in the form of a foliation into the $\theta_{(\ell)}=0$ spacelike twosurfaces and

2. there is no restriction on the signature of $H$ and so it can be spacelike, null, timelike, or conceivably some mixture of the three.

We label the foliation two-surfaces $S_{v}$, where $v$ is a foliation parameter. Furthermore, we assume that $S_{1} \equiv \Sigma_{1} \cap H$ and $S_{2} \equiv \Sigma_{2} \cap H$ are the initial and final leaves of that foliation. That is, $S_{1} \equiv S_{v_{1}}$ and $S_{2} \equiv S_{v_{2}}$ and $v_{1} \leq v \leq v_{2}$. The foliation parameter naturally induces the one-form $d v \in T^{*} H$ normal to the horizon 2-surfaces. In addition, we introduce a vector field $\frac{\partial}{\partial v}$ tangent to the horizon and satisfying $\mathcal{L}_{(\partial / \partial v)} v=1$. It is not unique, but will be useful in describing how the cross-sections $S_{v}$ evolve.

Since the horizon is foliated, we can introduce normals to the two-surfaces. It is most convenient to work with the null normals $\ell_{a}$ and $n_{a}$ to the surfaces $S_{v}$. This will allow us to treat timelike, spacelike and null horizons $H$ together and also make it easier to impose the condition that $\theta_{(\ell)}=0$. As shown in figure 2, we let $\ell^{a}$ be outward (towards $B$ ) and future pointing while $n^{a}$ will be inward (away from $B$ ) and also future pointing. Furthermore, we require the standard normalization $\ell \cdot n=-1$. This, however, still leaves one rescaling degree of freedom in the null vectors which will be important during the calculations. Irrespective of how this is chosen, the two-metric $\tilde{q}_{A B}$ satisfies $\tilde{q}^{a b}=p_{A}{ }^{a} p_{B}^{b} \tilde{q}^{A B}=g^{a b}+\ell^{a} n^{b}+n^{a} \ell^{b}$. As on the other two-surfaces, the metric compatible derivative will be denoted $d_{A}$. Additionally, in Section IB we have introduced the extrinsic curvatures $k_{(\ell)}^{A B}$ and $k_{(n)}^{A B}$ of the null vectors, as well as the one form $\tilde{\omega}_{A}$ which contains the angular momentum information.

On the initial and final cross sections of the horizon, we can relate the null normals $\ell$ and $n$ to those inherited from $\Sigma_{1}$ and $\Sigma_{2}$. Specifically, we have the timelike normal $\hat{u}^{a}$ inherited from the $\Sigma_{1} / \Sigma_{2}$ boundaries and the outward-pointing spacelike normal $\hat{n}^{a}$ to $S_{1} / S_{2}$ in $\Sigma_{1} / \Sigma_{2}$ as shown in figure 2] Then

$$
\ell^{a}=\zeta\left(\hat{u}^{a}+\hat{n}^{a}\right) \quad \text { and } \quad n^{a}=\frac{1}{2 \zeta}\left(\hat{u}^{a}-\hat{n}^{a}\right)
$$

for some function $\zeta$. Furthermore, we can also write the two-metric as $\tilde{q}^{a b}=g^{a b}+\hat{u}^{a} \hat{u}^{b}-\hat{n}^{a} \hat{n}^{b}$.

We now turn to characterizing the three-dimensional horizon $H$. We begin by introducing a normal to $H$ in $M$, which we denote $\tau_{a}$, and a vector field $\mathcal{V}^{a}$ that is both tangent to $H$ (so 
that $\mathcal{V}^{a} \tau_{a}=0$ ) and perpendicular to the $S_{v}$. Both are assumed to be future pointing (in the sense that $\mathcal{V} \cdot n$ and $\tau \cdot n$ are strictly negative). Since the horizon is not of fixed signature, there is no natural normalization for these structures, however, it will often be convenient to normalize both relative to the foliation label $v$. Thus, we require

$$
\mathcal{L}_{\mathcal{V}} v=1 \Leftrightarrow \frac{\partial}{\partial v}=\mathcal{V}+\tilde{V} \quad \text { where } \quad \tilde{V} \in T S_{v}
$$

and furthermore,

$$
\tau \cdot \tau=-\mathcal{V} \cdot \mathcal{V}
$$

For those familiar with dynamical horizons [9], when $H$ is spacelike, $\mathcal{V}^{a}$ is spacelike and proportional to the unit vector $\hat{r}^{a}$ of those papers. Similarly $\tau_{a}$ becomes timelike and is proportional to the unit $\hat{\tau}_{a}$ of those papers. By contrast, when $H$ is null, the fields are degenerate and are both parallel to $\ell$.

For a given two-surface $S_{v}$, we have introduced two sets of (future-pointing) normal vector fields: $\left(\ell^{a}, n^{a}\right)$ and $\left(\mathcal{V}^{a}, \tau^{a}\right)$. Clearly these must be related. To this end, we introduce a scalar field $C$ on $H$ chosen so that $\ell_{a}+C n_{a}$ is normal to $H$, and hence proportional to $\tau_{a}$. It follows that $\ell^{a}-C n^{a}$ will then lie in $H$ and be proportional to $\mathcal{V}^{a}$. Therefore, we can write

$$
\mathcal{V}^{a}=\alpha\left(\ell^{a}-C n^{a}\right) \text { and } \tau_{a}=\alpha\left(\ell_{a}+C n_{a}\right),
$$

for some $\alpha>0$ on $H$. The sign of $C$ naturally relates to the signature of the induced metric $q_{i j}=p_{i}{ }^{a} p_{j}{ }^{b} g_{a b}$ on $H$; specifically, if $C<0, C=0$, or $C>0, H$ is respectively timelike, null, or spacelike. Finally, the orientation of the volume form on the horizon is chosen so that

$$
\epsilon=\tau \wedge \mathrm{d} v \wedge \widetilde{\epsilon}=-\ell \wedge \boldsymbol{n} \wedge \widetilde{\boldsymbol{\epsilon}}
$$

Note that we have said very little about the geometry of $H$ itself. The reason for this is that the ensuing calculations will largely focus on two-surface rather than three-dimensional quantities. This is the most convenient way to proceed given that $H$ does not have a fixed signature and so may change between spacelike, null, and timelike signatures. Similarly, in order to avoid having to deal with the timelike, null, and spacelike quantities seperately we choose not to work with normalized quantities such as $\hat{\tau}_{a}$ and $\hat{r}^{a}$ which are ill-defined if $H$ becomes null.

\section{B. Boundary conditions}

In order to obtain a well-defined action principle, it is necessary to specify boundary conditions for all of the boundary manifolds presented in the previous section. First, on the initial and final boundaries $\Sigma_{1}$ and $\Sigma_{2}$, as well as the outer boundary $B$, we fix the three metric. Similarly on the two-dimensional intersections $\mathcal{B}$, we fix the two-metric. Thus, we require that

$$
\delta h_{i j}=0, \quad \delta \gamma_{i j}=0 \quad \text { and } \quad \delta \bar{\gamma}_{A B}=0 .
$$

These are by no means the only permissible boundary conditions, but they are by far the most common. See [12] for a discussion of alternative boundary conditions.

The situation on $H$ is more complicated and consequently more interesting. We start with a defining condition, $\theta_{(\ell)}=0$, and so expect it to play an important role in any calculations

- otherwise the resulting action formulation will in no way be tied to horizons. With this 
principle in mind we reject the idea of fixing the full three-metric $q_{i j}$ as this would give a well-defined action principle regardless of the value of $\theta_{(\ell)}$. Geometrically, it would also seem to be overly profligate compared to what is done on the other boundaries. For example, for $\Sigma_{1}$, the geometry is fully specified by its intrinsic metric, $h_{i j}$, and extrinsic curvature, $K_{(\hat{u})}^{i j}$. Each of these has six components, so for $\Sigma_{1}$ with standard boundary conditions, six out of a possible twelve geometric components are fixed. Now, if we fix $q_{i j}$ on $H$ as well as $\theta_{(\ell)}=0$ we would essentially have fixed seven of those components. By contrast, the path that we follow in this paper will fix $\theta_{(\ell)}=0$ along with just five other components of the surface geometry on $H$, and each of those conditions will play a crucial role in the calculations.

$H$ is also different from the other boundaries in that it comes with a foliation. This foliation is not just an optional structure imposed on $H$, but is crucial to its very definition. Without the foliation, the key $\theta_{(\ell)}=0$ condition is not well defined and, furthermore, for a given (spacelike) $H$ this foliation is unique [25]. Given this intricate association of $H$ and its foliation, it is perhaps not surprising that it also features prominently in our boundary conditions on $H$. We fix:

1. $\theta_{(\ell)}=0$,

2. the spacelike two-metric $\tilde{q}_{A B}$ on each $S_{v}$ (which also fixes $\widetilde{\boldsymbol{\epsilon}}$ ),

3. $\tilde{\omega}_{A}=-p_{A}{ }^{a} n_{b} \nabla_{a} \ell^{b}$ (the connection on the normal bundle to each $S_{v}$ ), and

4. $\ell_{i}=p_{i}{ }^{a} \ell_{a}$, the pull-back of $\ell_{a}$ to $T^{*} H$.

Then in the action formulation, the first variation of each of these quantities is zero. The foliation surfaces $S_{v}$ as well as their coordinate label $v$ are also kept fixed under these variations.

The first condition is simply one of the defining conditions for a trapping or dynamical horizon. Note however, that other defining conditions such as $\theta_{(n)}<0, \mathcal{L}_{n} \theta_{(\ell)}<0$, or $H$ being spacelike, are not imposed. Thus, these boundary conditions apply equally well to dynamical horizons or any of the varieties of trapping horizons, including those usually taken to represent white holes or inner black hole horizons [6]. It also includes three-dimensional surfaces traced by smoothly evolving apparent horizons. ${ }^{7}$

The second condition fixes the intrinsic geometry of each of the $S_{v}$ two-surfaces and so is somewhat similar to the usual fixing of the boundary metric. The difference, of course, is that here only the two-geometries are fixed. The rest of the three-geometry which gives the details how the two-surfaces evolve into each other, including the signature of $H$, is left free. Thus, while we know quantities such as the curvature scalar and area of the two-surfaces, the boundary conditions won't fix such things as the shear of $H$, or indeed any other locally defined rate of change.

The third condition fixes another part of the extrinsic geometry of $S_{v}$ by specifying the connection on the normal bundle. Equivalently, it may be thought of as fixing the angular momentum of the horizon. Combined with the previous conditions, we see that the boundary

\footnotetext{
${ }^{7}$ However, we do not allow for situations where $\mathcal{L}_{n} \theta_{(\ell)}$ changes sign and so $C$ diverges, such as the spacetimes considered in 24]. These situations can be dealt with fairly easily by sectioning the horizon into spacelike/timelike sections over which $C$ is well-defined, but for simplicity we will not consider these cases here.
} 
conditions on $H$ are mixed in the sense that they fix parts of both the intrinsic and extrinsic (two) geometry of the $S_{v}$. This contrasts with the conditions on $B$ and $\Sigma_{1,2}$ which exclusively fixed the intrinsic (three) geometry. It is interesting to note that Epp [27] presented counting arguments which suggest that knowledge of the intrinsic geometry of a two-surface plus the curvature on its normal bundle should be sufficient to uniquely determine its embedding in a four-dimensional space of constant curvature. Thus, in this sense, the information that we have specified on $S_{v}$ can be thought of as a complete set of data that fully specifies its geometry.

Finally, we come to the fourth term. At first glance it may appear that we are fixing a seventh "component" of the geometry (given that $\theta_{(\ell)}=0$ is one, $\tilde{q}_{A B}$ gives three, and $\tilde{\omega}_{A}$ gives two). However, that is not the case. Recall that when defining $\ell_{a}$ we left its normalization (and so that of $n_{a}$ ) unspecified. This condition removes that degree of freedom. Hence, it is essentially equivalent to a requirement like the one that specifies that extrinsic curvatures should be defined using unit-normal vectors to surfaces. Just as such a definition is necessary so that a discussion of extrinsic curvature is meaningful, here we need a fix to give $\tilde{\omega}_{A}$ a geometric meaning — otherwise it would change with rescalings of $\ell_{a}$. Keep in mind that we only fix the pullback of $\ell$ to $H$, not $\ell_{a}$ itself (which would have geometric significance).

\section{The quasilocal action and its variation}

Having defined $M$ and established this notation, we turn to the action itself. The action for the region $M$ and its boundaries is given by

$$
\begin{aligned}
& I_{H}=\frac{1}{16 \pi G} \int_{M} \boldsymbol{\epsilon \mathcal { R }}+\frac{1}{8 \pi G} \int_{\Sigma_{2}-\Sigma_{1}}^{(\Sigma)} \boldsymbol{\epsilon} K_{\hat{u}}
\end{aligned}
$$

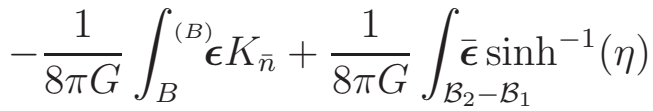

$$
\begin{aligned}
& +\frac{1}{8 \pi G} \int_{H} \tilde{\boldsymbol{\epsilon}} \wedge d v\left(\kappa_{\mathcal{V}}+\tilde{V}^{A} \tilde{\omega}_{A}\right)-\frac{1}{8 \pi G} \int_{S_{2}-S_{1}} \tilde{\boldsymbol{l}} \ln \zeta,
\end{aligned}
$$

where $\mathcal{R}$ is the Ricci scalar over $M, \kappa_{\mathcal{V}}$ is given by

$$
\kappa_{\mathcal{V}}=\mathcal{V}^{a} \omega_{a}=-\mathcal{V}^{a} n_{b} \nabla_{a} \ell^{b}
$$

and $\tilde{V}^{a}$ is defined in (3.3). Equivalently, $\kappa_{\mathcal{V}}+\tilde{V}^{A} \tilde{\omega}_{A}=\left(\frac{\partial}{\partial v}\right)^{i} \omega_{i}$, and so we see that this action explicitly depends on both the foliation and the associated vector field $\left(\frac{\partial}{\partial v}\right)$ (though in the upcoming calculations, we will see that this is essentially a gauge dependence only as the variation of $I_{H}$ will vanish whatever the value of these coordinate quantities).

The bulk, $\Sigma, B$, and $\mathcal{B}$ boundary terms are standard and found in all discussions of the gravitational action. The integral of the Ricci scalar over $M$ is the Einstein-Hilbert bulk term while the integrals over $\Sigma_{1}, \Sigma_{2}$, and $B$ are the usual extrinsic curvature terms which must be included when $M$ has timelike/spacelike boundaries (see, for example, [16]). The fourth term may be a little less familiar, but is also well known [28]. This "corner" term is necessary when the $\Sigma$ boundaries do not orthogonally intersect $B$; that is $\hat{u}^{a} \bar{n}_{a} \neq 0$. The final two terms arise at the horizon boundary and so are new, though we immediately note that the last is a "corner" term between $H$ and $\Sigma_{1,2}$. 
Evaluating the first variation of this metric is a non-trivial calculation. Most readers will not be interested in the details and so they are relegated to Appendix A. Here, we just quote the final result:

$$
\begin{aligned}
& \delta I_{H}=\frac{1}{16 \pi G} \int_{M} \boldsymbol{\epsilon} G_{a b} \delta g^{a b}-\frac{1}{16 \pi G} \int_{\Sigma_{2}-\Sigma_{1}}^{(\Sigma)}\left(K_{\hat{u}}^{i j}-K_{\hat{u}} h^{i j}\right) \delta h_{i j} \\
& +\frac{1}{16 \pi G} \int_{B}^{(B)} \boldsymbol{\epsilon}\left(K_{\bar{n}}^{i j}-K_{\bar{n}} \gamma^{i j}\right) \delta \gamma_{i j}+\frac{1}{8 \pi G} \int_{\mathcal{B}_{2}-\mathcal{B}_{1}}(\delta \overline{\boldsymbol{c}}) \sinh ^{-1}(\eta) \\
& +\frac{1}{16 \pi G} \int_{H} \mathbf{d} \boldsymbol{v} \wedge \widetilde{\boldsymbol{\epsilon}}\left(\tilde{s}^{A B} \delta \tilde{q}_{A B}\right)+\frac{1}{8 \pi G} \int_{S_{2}-S_{1}} \delta \widetilde{\boldsymbol{\epsilon}}(1-\ln \zeta) \\
& +\frac{1}{8 \pi G} \int_{H}\left\{\tilde{\boldsymbol{\epsilon}} \wedge\left((\delta \underline{\ell}) \theta_{(n)}-(\delta \underline{\boldsymbol{n}}) \theta_{(\ell)}\right)+\tilde{V}^{A} \delta\left(\mathbf{d} \boldsymbol{v} \wedge \tilde{\boldsymbol{\epsilon}}_{\tilde{\omega}_{A}}\right)-\delta\left(2 \theta_{(\ell)} \boldsymbol{\boldsymbol { n }} \wedge \tilde{\boldsymbol{\epsilon}}\right)\right\} .
\end{aligned}
$$

Here, the quantity $\tilde{s}^{A B}$ is given by

$$
\tilde{s}^{A B}=\left(k_{\tau}+\kappa \mathcal{V}\right) \tilde{q}^{A B}-k_{\tau}^{A B},
$$

while $\underline{\ell}$ and $\boldsymbol{n}$ respectively denote the pull-backs of $\ell_{a}$ and $n_{a}$ into $H$.

As for the action itself, the boundary terms on $\Sigma_{1,2}$ and $\mathcal{B}_{1,2}$ are completely standard and it is clear that fixing $\delta h_{i j}=0$ and $\delta \gamma_{i j}=0$ will ensure that these vanish. Similarly, it is not hard to see that our boundary conditions from Section 1 IIB are sufficient to ensure the terms on $H$ and $S_{1,2}$ vanish. Thus, $\delta I_{H}=0$ if and only if Einstein's equations hold in the bulk and so this action is well-formulated.

Finally we note that, as in most Lagrangian analyses, the action that we have considered is not the only action compatible with the given boundary conditions. In fact, any functional of the form

$$
I_{H}^{\prime}=I_{H}+\text { (functional of the fixed data), }
$$

will work equally well, since the variation of a functional of the fixed data is zero. The implications of this freedom will be discussed in more detail in Section $11 \mathrm{E}$

\section{Weak boundary conditions}

In the previous section, we have seen that the action principle is well-formulated for a manifold with a trapping horizon boundary. In the calculation, the boundary conditions have been imposed in the standard way; namely by requiring that variations of fixed quantities vanish. However for boundary conditions such as those on $B$, it has recently been shown [14, 15] that a weaker form of the boundary conditions can be used instead. These weak boundary conditions only fix the data up to diffeomorphisms. The motivation for this is the principle that physical theories should be generally covariant. In the presence of boundaries, we take this to mean that while we may (partially) specify the geometry of those surfaces, we should not tie that geometry to specific points of $\Sigma_{1,2}, \mathcal{B}_{1,2}, S_{1,2}, B$ or $H$ - just as in general relativity spacetime geometry isn't tied to points of an underlying manifold (or equivalently a coordinate system).

We now show that the boundary conditions of Section IIIB can also be applied in this weak form - up to diffeomorphisms which preserve the underlying manifold structure of the boundaries. Therefore, we require that

$$
\delta h_{i j}=\mathcal{L}_{Y} h_{i j}, \quad \delta \gamma_{i j}=\mathcal{L}_{Z} \gamma_{i j} \quad \text { and } \quad \delta \bar{\gamma}_{A B}=\mathcal{L}_{\bar{y}} \bar{\gamma}_{A B}
$$


where $Y^{i} \in T \Sigma_{1,2}, Z^{i} \in T B$ and $\bar{y}^{A} \in T \mathcal{B}_{1,2}$. Since the boundaries $\mathcal{B}_{1,2}$ must be preserved (and so mapped into themselves), we impose the further restrictions that

$$
Y^{a}=Z^{a}=\bar{y}^{a} \text { on } \mathcal{B}_{1,2},
$$

where each of these is the push-forward of the appropriate lower-dimensional vector field.

Similarly, at the horizon, we allow diffeomorphisms which preserve the geometric structure, which in this case includes the foliation $S_{v}$. Thus, such maps should send all points in a given leaf $S_{v}$ into a second leaf $S_{\phi_{X}(v)}$ for some function $\phi_{X}(v):\left[v_{1}, v_{2}\right] \rightarrow\left[v_{1}, v_{2}\right]$. The most general vector fields generating a one-parameter family of diffeomorphisms of this type take the form

$$
X^{i}=x_{o} \mathcal{V}^{i}+p_{A}{ }^{i} \tilde{x}^{A} \text { where } \tilde{x}^{A} \in T S_{v},
$$

where $x_{o}=x_{o}(v)$ is a function of the foliation parameter $v$, and

$$
x_{o}=0 \text { and } Y^{i}=\tilde{x}^{i} \text { on } S_{1,2} .
$$

Then, the weak form of the boundary conditions fixes :

$$
\delta \tilde{q}_{A B}=\mathcal{L}_{X} \tilde{q}_{A B}, \quad \delta \tilde{\omega}_{A}=\mathcal{L}_{X} \tilde{\omega}_{A}, \quad \delta \underline{\leftarrow}_{i}=\mathcal{L}_{X} \underline{\ell}_{i} \quad \text { and } \quad \theta_{(\ell)}=\delta \theta_{(\ell)}=0 .
$$

The action principle will remain well-defined if we also enforce the diffeomorphism constraint on each of the boundary three-surfaces. This is not a big assumption as these are naturally induced if the Einstein equations hold in the bulk. Given a normal $\chi_{a}$ and a tangent vector $X^{a}$ to a surface, the (vacuum) diffeomorphism constraint on that surface is $X^{a} G_{a}{ }^{b} \chi_{b}=0$. Then on $\Sigma_{1,2}$ and $B$ this constraint says that

$$
D_{i}\left(K_{\hat{u}}^{i j}-K_{\hat{u}} h^{i j}\right)=0 \quad \text { and } \quad \Delta_{i}\left(K_{\bar{n}}^{i j}-K_{\bar{n}} \gamma^{i j}\right)=0,
$$

where we respectively take $\hat{u}$ and $\hat{n}$ as the normal vectors.

The situation on $H$ is a little more complicated since its uncertain signature means that we cannot utilize unit normals or assume the existence of a (unique) inverse metric. The most useful form for our purposes is found by taking $\tau_{a}$ (defined in equation (3.5)) as the normal and evaluating

$$
X^{a} G_{a}{ }^{b} \tau_{b}=0
$$

where we continue to assume that $T_{a b}=0$. For the current calculation we further specialize this by integrating over $S_{v}$. Then, as noted in [10]:

$$
\frac{1}{8 \pi G} \int_{S_{v}}\left\{\kappa_{\mathcal{V}} \mathcal{L}_{X} \widetilde{\boldsymbol{\epsilon}}+\tilde{x}^{A} \mathcal{L}_{\mathcal{V}}\left[\widetilde{\boldsymbol{\epsilon}} \tilde{\omega}_{A}\right]\right\}=\frac{1}{16 \pi G} \int_{S_{v}} \widetilde{\boldsymbol{\epsilon}}\left\{k_{\tau}^{A B} \mathcal{L}_{X} \tilde{q}_{A B}+2 \alpha C \mathcal{L}_{X} \theta_{(n)}\right\}
$$

A full derivation of this result will be found in [29], but for now a few lines of algebra puts it into a more useful form for the current calculation:

$$
\frac{1}{8 \pi G} \int_{S_{2}-S_{1}} \tilde{\boldsymbol{\epsilon}}\left(\tilde{\omega}_{A}^{A}\right)=\frac{1}{8 \pi G} \int_{H}\left\{\mathbf{d} \boldsymbol{v} \wedge \widetilde{\boldsymbol{\epsilon}}\left(\frac{\tilde{s}^{A B}}{2} \mathcal{L}_{X} \tilde{q}_{A B}\right)-\tilde{\boldsymbol{\epsilon}} \wedge\left(\mathcal{L}_{X} \underset{\leftarrow}{\ell}\right) \theta_{(n)}-\tilde{V}^{A} \mathcal{L}_{X}\left(\mathbf{d} \boldsymbol{v} \wedge \tilde{\boldsymbol{\epsilon}}_{\tilde{\omega}}\right)\right\} .
$$


With the weaker boundary conditions, the variation of the action (3.10) becomes

$$
\begin{aligned}
& \delta I_{H}=\frac{1}{16 \pi G} \int_{M} \boldsymbol{\epsilon} G_{a b} \delta g^{a b}-\frac{1}{16 \pi G} \int_{\Sigma_{2}-\Sigma_{1}}^{(\Sigma)} \boldsymbol{\epsilon}\left(K_{\hat{u}}^{i j}-K_{\hat{u}} h^{i j}\right) \mathcal{L}_{Y} h_{i j} \\
& +\frac{1}{16 \pi G} \int_{B}^{\left({ }^{(B)} \boldsymbol{\epsilon}\right.}\left(K_{\bar{n}}^{i j}-K_{\bar{n}} \gamma^{i j}\right) \mathcal{L}_{Z} \gamma_{i j}+\frac{1}{8 \pi G} \int_{\mathcal{B}_{2}-\mathcal{B}_{1}}\left(\mathcal{L}_{y} \overline{\boldsymbol{\epsilon}}\right) \sinh ^{-1}(\eta) \\
& +\frac{1}{16 \pi G} \int_{H} \mathbf{d} \boldsymbol{v} \wedge \widetilde{\boldsymbol{\epsilon}}\left(\tilde{s}^{A B} \mathcal{L}_{X} \tilde{q}_{A B}\right)+\frac{1}{8 \pi G} \int_{S_{2}-S_{1}} \mathcal{L}_{\tilde{x}} \tilde{\boldsymbol{\epsilon}}(1-\ln \zeta) \\
& +\frac{1}{8 \pi G} \int_{H}\left\{\tilde{\boldsymbol{\epsilon}} \wedge\left(\left(\mathcal{L}_{X} \underset{\leftarrow}{\ell}\right) \theta_{(n)}-(\delta \underline{\boldsymbol{n}}) \theta_{(\ell)}\right)+\tilde{V}^{A} \mathcal{L}_{X}\left(\mathbf{d} \boldsymbol{v} \wedge \tilde{\boldsymbol{\epsilon}}_{\tilde{\omega}_{A}}\right)-\delta\left(2 \underline{\boldsymbol{n}} \theta_{(\ell)} \wedge \tilde{\boldsymbol{\epsilon}}\right)\right\} .
\end{aligned}
$$

The diffeomorphism constraints of $\Sigma_{1,2}$ and $B$ allow us to rewrite the boundary terms on those surfaces as exact derivatives. These can then be integrated to give contributions on $\mathcal{B}_{1,2}$ and $S_{1,2}$. Similarly, the horizon constraint (3.21) can be used to replace the terms on $H$ with a term on $S_{1,2}$. Therefore, we arrive at

$$
\begin{gathered}
\delta I_{H}=\frac{1}{16 \pi G} \int_{M} \boldsymbol{\epsilon} G_{a b} \delta g^{a b}+\frac{1}{8 \pi G} \int_{\mathcal{B}_{2}-\mathcal{B}_{1}}\left\{\left(\mathcal{L}_{y} \overline{\boldsymbol{\epsilon}}\right) \sinh ^{-1}(\eta)-\overline{\boldsymbol{\epsilon}} y^{a}\left(\hat{n}^{b} \nabla_{a} \hat{u}_{b}+\bar{u}^{b} \nabla_{a} \bar{n}_{b}\right)\right\} \\
+\frac{1}{8 \pi G} \int_{S_{2}-S_{1}}\left\{\left(\mathcal{L}_{\tilde{x}} \widetilde{\boldsymbol{\epsilon}}\right)(1-\ln \zeta)+\widetilde{\boldsymbol{\epsilon}} \tilde{x}^{a}\left(\hat{n}^{b} \nabla_{a} \hat{u}_{b}+\bar{n}^{b} \nabla_{a} \bar{u}_{b}\right)\right\}
\end{gathered}
$$

Finally, we can make use of the relationship between $\eta, \hat{n}, \hat{u}$ and $\bar{n}, \bar{u}$ given in (3.1) to show that the terms on $\mathcal{B}_{1,2}$ evaluate to an exact derivative which then integrates to zero. Similarly the terms on $S_{1,2}$ are exact as can easily be seen by using the relationship between $\zeta, \hat{n}, \hat{u}$ and $\ell, n$ given in (3.2). The only remaining term is then the bulk term which vanishes for solutions to Einstein's equations. Thus, we have shown that the action principle remains well formulated when we enforce weak boundary conditions while requiring that the diffeomorphism constraints hold on the boundaries of the spacetime.

With these weak boundary conditions, the freedom in the action is vastly reduced as boundary terms like those of (3.12) must now be covariantly defined. That said, it is clear that the parameter space of possible actions remains large and, for example, includes all actions of the form

$$
I_{H}^{\prime}=I_{H}+\int_{\Sigma_{2}-\Sigma_{1}} \mathbf{F}^{\Sigma}\left(h_{i j}\right)+\int_{B} \mathbf{F}^{B}\left(\gamma_{i j}\right)+\int_{H} \mathbf{F}^{H}\left(\tilde{q}_{A B}, \tilde{\omega}_{A}, \underline{\ell}, \theta_{(\ell)}, v\right),
$$

where the $\mathbf{F}$ are required to be dimensionally correct, but are otherwise freely defined volume forms with the displayed functional dependencies. A lot has been written on possible boundary terms on $B$ (see for example [11, 27, 30]) but for now we simply note that the mathematical formalism allows these freedoms and that they can only be further reduced by imposing extra restrictions on the system. A good example of such a restriction is the requirement that the evaluated action not diverge in asymptotically flat spacetimes as $B$ is taken to infinity. In this case extra boundary terms on $B$ not only may, but in fact must be added to the action to remove such divergences.

In this paper, the external boundary $B$ is not the chief concern and so we will not consider these terms further. Instead we focus on the internal boundary $H$ and so consider actions that are equivalent to $I_{H}$ up to terms of the form

$$
\Delta I=-\int d v E\left[\tilde{q}, \tilde{\omega}, \underset{\leftarrow}{\ell}, \theta_{(\ell)}, v\right]
$$




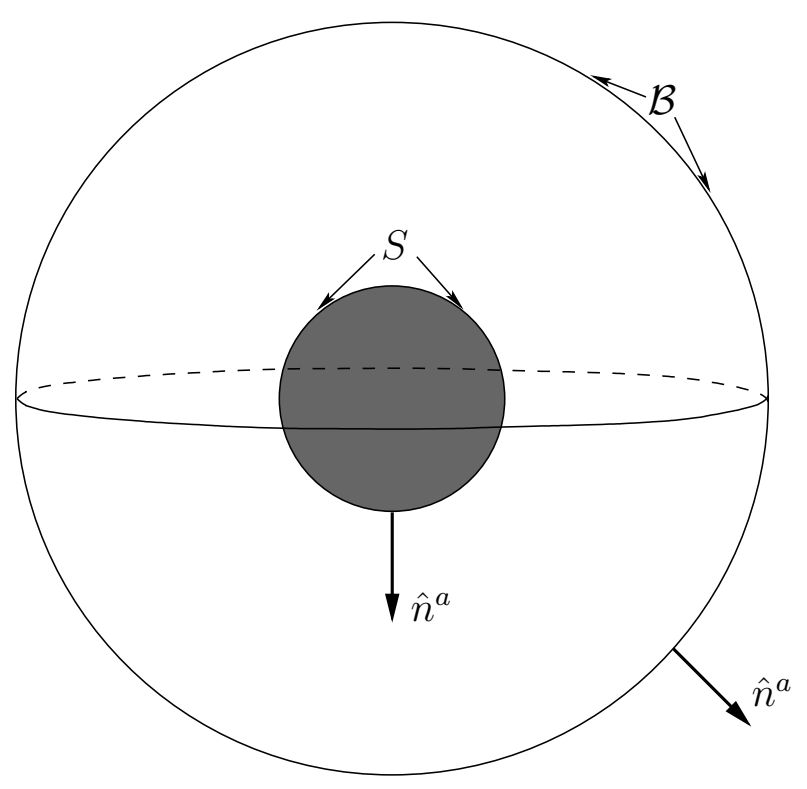

FIG. 3: A three-dimensional volume $\Sigma_{t}$ along with its boundaries and their normals.

where $E\left[\tilde{q}, \tilde{\omega}, \underline{\ell}, \theta_{(\ell)}, v\right]$ is a free functional of the fixed data with dimension length. The requirement that the extra boundary term break up in this way is natural given the primary role played by the foliation of $H$. As we will now see, such terms are also very convenient as they allow for a clean Legendre transform of the action.

\section{E. Legendre transform to obtain a Hamiltonian}

In the previous sections it has been shown that the action $I_{H}$ (3.8) is valid with either a strong or a weak fixing of the boundary conditions. In this section we introduce a notion of time to $M$ which then allows us to perform a Legendre transform on the action and so derive a corresponding Hamiltonian functional.

Flow of time and associated quantities

Coordinate time must be externally imposed in general relativity. We do this in the following manner. First, foliate $M$ with spacelike (partial Cauchy) surfaces $\left\{\left(\Sigma_{t}, h_{i j}\right) ; t_{1} \leq t \leq t_{2}\right\}$; these will correspond to "instants of simultaneity" (see figure 3). For simplicity, we assume that this foliation is compatible with all of the structures that we have already introduced. Thus $\Sigma_{t_{1,2}}=\Sigma_{1,2}$ and if $\left\{\mathcal{B}_{t} ; t_{1} \leq t \leq t_{2}\right\}$ is the induced foliation of $B$, then $\mathcal{B}_{t_{1,2}}=\mathcal{B}_{1,2}$. On $H$ we require that the induced foliation be compatible with $S_{v}$ and so it is natural to drop $v$ and henceforth use the single parameter $t$ to characterize both foliations. This extra structure on $M$ and its boundaries allows us to extend the definitions of several quantities that were previously only defined on initial and final boundaries $\mathcal{B}_{1,2}$ and $S_{1,2}$. Specifically, with the help of $\Sigma_{t}$ we can define $\hat{u}^{a}$ and $\hat{n}^{a}$ everywhere on $B$ and $H$. Further $\bar{u}^{a}$ and $\eta:=-\hat{u} \cdot \bar{n}$ may be extended over $B$, while $\zeta$ may be defined everywhere on $H$.

In addition to a foliation parameter, we must also introduce a corresponding notion of evolution. This is provided by the evolution vector field $T^{a}$ which is assumed to be 
compatible with the foliation in the sense that

$$
\mathcal{L}_{T} t=1 .
$$

Thus, the flow generated by $T^{a}$ evolves leaves of the foliation into each other in the required way. It is also assumed to be compatible with the boundaries so it maps them into themselves; equivalently on $B / H, T^{a} \in T B / H$ respectively. Then, on $H$ it is natural to choose $\left(\frac{\partial}{\partial v}\right)^{a}:=T^{a}$. Note that nowhere in this construction have we assumed that $T^{a}$ be everywhere timelike. Just as the natural (Killing) evolution vector field for the Kerr spacetime is not everywhere causal (even outside the event horizon), so in this case $T^{a}$ is allowed to be null or spacelike. Regardless of the nature of $T^{a}$, it can be decomposed into components normal and tangent to the three-surfaces $\Sigma_{t}$ as

$$
T^{a}=N \hat{u}^{a}+V^{a},
$$

where $N$ is the lapse function and $V^{a} \in T \Sigma_{t}$ is the shift vector field. Given that $T^{a}$ generates $B$ and $H$ we can perform similar decompositions on those surfaces. Respectively we have

$$
\begin{aligned}
T^{a} & =\bar{N} \bar{u}^{a}+\bar{V}^{a} \quad \text { and } \\
T^{a} & =\left(\frac{\partial}{\partial v}\right)^{a}=\mathcal{V}^{a}+\tilde{V}^{a},
\end{aligned}
$$

where $\bar{N}=-T \cdot \bar{u}, V^{a}=p_{A}{ }^{a} \bar{V}^{A}$ on $\mathcal{B}_{t}$ and $V^{a}=p_{A}{ }^{a} \tilde{V}^{A}$ on $S_{t}$. Finally, the foliation parameter can be used to relate the various volume forms. Specifically, $\hat{u}_{a}=-N \nabla_{a} t$, so

$\boldsymbol{\epsilon}=-N \mathbf{d t} \mathbf{\wedge} \wedge^{(\Sigma)} \boldsymbol{\epsilon}$. Similarly, on boundaries we have ${ }^{(B)} \boldsymbol{\epsilon}=\bar{N} \mathbf{d t} \wedge \overline{\boldsymbol{\epsilon}}$ and ${ }^{(H)} \boldsymbol{\epsilon}=\mathbf{d t} \wedge \tilde{\boldsymbol{\epsilon}}$, where $\underline{\mathbf{d t}}$ is the appropriate pullback of $\mathbf{d t}$.

\section{Legendre transform}

Using this foliation, we can break up the action into space and time components in the standard way [16]. To this end, we introduce the momentum $P^{a b}$ conjugate to $h_{a b}$ as

$$
P^{a b}=\frac{1}{16 \pi G}\left(K_{\hat{u}}^{a b}-K_{\hat{u}} h^{a b}\right) .
$$

Then, it is straightforward to show that

$$
\frac{1}{16 \pi G} N \mathcal{R}=N \mathcal{H}+V^{a} \mathcal{H}_{a}+P^{a b} \mathcal{L}_{T} h_{a b}-2 \mathcal{L}_{T} K_{\hat{u}}-K_{\hat{u}} h^{a b} \mathcal{L}_{T} h_{a b}+2 D_{a}\left(h^{a b} T^{c} \nabla_{c} \hat{u}_{b}\right)
$$

where $\mathcal{H}$ and $\mathcal{H}_{a}$ are the usual Hamiltonian and diffeomorphism constraints,

$$
\begin{aligned}
\mathcal{H} & =\frac{R}{16 \pi G}-16 \pi G\left(P^{a b} P_{a b}-P^{2} / 2\right) \quad \text { and } \\
\mathcal{H}_{a} & =2 D_{b} P_{a}{ }^{b}
\end{aligned}
$$

which vanish on shell.

We can then substitute equation (3.31) into our expression for the action (3.8) and make use of the relationships between the various normals at $\mathcal{B}_{t}$ and $S_{t}$ given in (3.1) and (3.2) to express the action as:

$$
I_{H}=\int-d t\left\{H(t)+\int_{\Sigma_{t}} \mathbf{P}^{i j} \mathcal{L}_{T} h_{i j}+\int_{S_{t}} \tilde{P} \mathcal{L}_{T} \tilde{\boldsymbol{\epsilon}}-\int_{\mathcal{B}_{t}} \bar{P} \mathcal{L}_{T} \overline{\boldsymbol{\epsilon}}\right\} .
$$


Here the Hamiltonian functional $H(t)$ is given by

$$
H(t)=\int_{\Sigma_{t}}^{(\Sigma)} \boldsymbol{\epsilon}\left(N \mathcal{H}+V^{i} \mathcal{H}_{i}\right)+\int_{\mathcal{B}_{t}}\left\{\frac{\overline{\boldsymbol{\epsilon}} \bar{N} k_{(\bar{n})}}{8 \pi G}+\overline{\boldsymbol{\epsilon}} \bar{V}^{A} \bar{\jmath}_{A}\right\},
$$

where

$$
\bar{\jmath}_{A}=\frac{p_{A}{ }^{a} \hat{u}^{b} \nabla_{a} \hat{n}_{b}}{8 \pi G}
$$

Furthermore,

$$
\mathbf{P}^{i j}:={ }^{(\Sigma)} \boldsymbol{\epsilon} P_{\hat{u}}^{i j}, \quad \bar{P}:=\frac{\sinh ^{-1} \eta}{8 \pi G} \text { and } \quad \tilde{P}:=\frac{\ln \zeta}{8 \pi G}
$$

are identified as the conjugate momenta to $h_{i j}, \widetilde{\boldsymbol{\epsilon}}$, and $\overline{\boldsymbol{\epsilon}}$ in the usual way [11, 13, 15, 31].

\section{The Hamiltonian functional}

Although it is conventional practice to refer to $H(t)$ as the Hamiltonian, it is clear that in many cases this functional is not really a Hamiltonian. The problem is that standard Hamiltonian mechanics properly applies only to closed systems. Here, however, energy flows are allowed through the boundaries $\mathcal{B}_{t}$ and $S_{t}$ and so in general $\dot{H}(t) \neq 0$ which in turn means that it cannot really be a Hamiltonian functional. Technically, the Legendre transform and Hamiltonian identification can only be correctly applied in those cases where the system is isolated - that is when $H$ is an isolated horizon 8] and $B$ is similarly disconnected from its surroundings (a sufficient condition is that $\mathcal{L}_{T} \gamma_{i j}=0$ ). However, in 11] Hamilton-Jacobi arguments were used to demonstrate that the $H(t)$ derived in this way can still be interpreted as the quasilocal energy associated with $\Sigma_{t}$ and its boundaries. These arguments are also supported by the extended phase space arguments of [15].

With this in mind, we can consider what $H(t)$ tells us about the quasilocal energy of the system. First, since the bulk terms of (3.35) are pure constraints, the non-vanishing contributions to the energy come exclusively from the boundary terms. Thus, it is conventional to identify the value of each boundary contribution to $H(t)$ as the energy of that boundary. In this case, the only such term appears on $\mathcal{B}_{t}$. This term was first identified in 11] and has been much discussed since then. Note, however, that the value of the boundary energy is not uniquely specified. At $B$ we are free to add any free functional of the boundary metric $\gamma_{a b}$ to the action and hence obtain a different value for the energy of $B$. Indeed, in many cases (such as the limit of $\mathcal{B}_{t}$ going to infinity in asymptotically flat spacetime) it is necessary to add such reference terms so that the energy of $B$ doesn't diverge.

Next, we turn our attention to the horizon boundary. With our choice of action, we do not obtain a contribution to the Hamiltonian at $H$. However, as with the outer boundary $B$, we are free to add any functional of the fixed data to the action thereby obtaining a different value for the horizon energy. As we saw in Section IIID this is a significant freedom. Specifically, if we impose the boundary conditions weakly, we can add any diffeomorphism invariant function of the fixed boundary data. The freedom (3.25) in the action gives rise to a corresponding freedom of

$$
\Delta H=E\left[\tilde{q}_{A B}, \tilde{\omega}_{A}, \underline{\ell}, \theta_{(\ell)}, v\right]
$$

in $H(t)$. Thus, as far as the mathematical formalism is concerned the contribution to the energy from $S_{t}$ may be any functional of the fixed data. We will return to a more thorough discussion of the energy associated with a horizon boundary in Section $\nabla$. 
First, however, we re-examine this problem from the canonical phase space point of view. This will both give us a new perspective on the energy calculation and also allow us to derive an expression for the angular momentum of trapping horizons.

\section{CANONICAL PHASE SPACE FORMULATION}

In the previous section we have seen that trapping horizons can act as boundaries in a quasilocal action formulation of general relativity. Here we will see that they are also suitable boundaries in a canonical phase space description of gravity. Apart from the value that this demonstration has in its own right, it also gives us another point of view on some of the issues that arose in the last section. In particular, it will provide further elucidation of the relationship between the Hamiltonian and the energy functional $H(t)$, and a sharpening of the discussion of the allowed freedom in that functional itself. Additionally, while the action calculation permitted us to investigate time evolution and hence obtain the quasilocal energy functional, this Hamiltonian investigation will allow us to consider more general evolutions of the boundary data and so also tell us about other "conserved" quantities. Specifically, we will be able to study angular momentum as conjugate to rotations of the boundary data.

The particular formalism that we will use was described by the current authors in a recent paper [15] and is designed to deal with situations, such as this one, where the energy of the quasilocal region is not expected to be conservered. Such systems cannot be dealt with using standard Hamiltonian methods which, by their very nature, are tailored to cases where energy is conserved. The methods that we will use are based on the generalized Hamiltonian formalisms of classical mechanics which are specifically designed to deal with situations like these where the energy isn't constant. These methods expand the phase space to include the time coordinate as a configuration variable (see for example [32]). Then, it is possible to construct a conserved Hamiltonian that generates time translations in this new, extended phase space. In this space, the energy of the system is no longer the value of this Hamiltonian, but instead is the value of the momentum that is canonically conjugate to time. In 15] these methods from classical mechanics were extended to quasilocal general relativity. ${ }^{8}$ Just as in classical mechanics, the time coordinate appears as a configuration variable and the energy as its conjugate momenta. However, for quasilocal general relativity, we also include coordinates parameterizing the two-boundaries in the configuration space. This allows us to determine the angular momenta of the boundaries, which are conjugate to rotations of the boundary data.

\section{A. Phase Space and Symplectic Structure}

The Hamiltonian approach to general relativity is based on a three-dimensional manifold $\Sigma$ and its attendant fields which evolve in time with respect to some time parameter $t$, rather than the usual four-dimensional spacetime. In the case of interest here, $\Sigma$ is diffeomorphic to $[0,1] \times S^{2}$ and has boundaries $\mathcal{B}$ and $S$ (which are each diffeomorphic to $S^{2}$ ), as was shown in figure 3. Over this region, the basic defining fields of three-dimensional, time-dependent general relativity are the three-metric $h_{i j}$ over $\Sigma$ and its canonically conjugate momentum

\footnotetext{
${ }^{8}$ Other applications of extended phase space ideas to general relativity include the papers listed in 33 .
} 
$\mathbf{P}^{i j}:={ }^{(\Sigma)} \boldsymbol{\epsilon} P^{i j}$. These variables contain all the information needed to resconstruct the full four-geometry at any time $t$ and, once we specify a lapse function $N$ and shift vector $V^{i}$, also define how that geometry evolves in time (Appendix $\mathrm{B}$ ).

There are also degrees of freedom on $\mathcal{B}$ and $S$ that determine how these boundaries evolve relative to $\Sigma$. To specify these evolutions it is sufficient to know the "orthogonal velocity" vector fields — that is $v_{\vdash}=(V \cdot \hat{n}) / N$. These degrees of freedom are controlled by the area forms $\overline{\boldsymbol{\epsilon}}$ and $\widetilde{\boldsymbol{\epsilon}}$ and their conjugate momenta $\bar{P}$ and $\tilde{P}$ which we defined in (3.37). The encoding of this information is easily seen for $\mathcal{B}$. There,

$$
N=\bar{N} \sqrt{1+\eta^{2}} \quad \text { and } \quad V^{i}=(\bar{N} \eta) \hat{n}^{i}+\bar{V}^{i}
$$

and so $v_{\vdash}=\eta / \sqrt{1+\eta^{2}}$ (recall that $\bar{P}$ is defined in terms of $\eta$ ). On $S$ the relation is a little more complicated, since there are two variables which combine to define the evolution; $C$ relates horizon evolution to the null vectors and then $\zeta$ ties those null vectors to $\Sigma$ and $S$. On $S$,

$$
N=\alpha\left(\zeta-\frac{C}{2 \zeta}\right) \quad \text { and } \quad V^{i}=\alpha\left(\zeta+\frac{C}{2 \zeta}\right) \hat{n}^{i}+\tilde{V}^{i}
$$

Then $v_{\vdash}=\left(2 \zeta^{2}+C\right) /\left(2 \zeta^{2}-C\right)$ and the evolution in $\Sigma$ is given by $\tilde{P}$ (defined in terms of $\zeta$ ) once we know $C$.

In addition to these configuration and momentum variables, in the extended phase space we include the time foliation label $t$ and spatial coordinates on both boundaries, $\tilde{\theta}^{\alpha}$ and $\bar{\theta}^{\alpha}$ (for $\alpha=1,2$ ), as configuration variables in order to allow for energy and angular momentum flows through those boundaries [15]. Along with these variables come their conjugate momenta $P_{t}$ (a scalar field) and $\overline{\mathbf{P}}_{\alpha}$ and $\tilde{\mathbf{P}}_{\alpha}$ (one-form valued volume forms), which are closely related to energy and angular momentum. We can specify the evolution equations of the configuration variables; they are simply:

$$
\frac{d}{d t} t=1, \frac{d}{d t} \bar{\theta}^{\alpha}=0 \text { and } \frac{d}{d t} \tilde{\theta}^{\alpha}=0 .
$$

The values of the conjugate momenta and their evolution equations are initially left free, however we will see that the necessity of Hamiltonian evolution will greatly reduce that freedom - and so generate candidate expressions for the energy and angular momentum associated with the boundary $\mathcal{B}$ and horizon $S$.

The allowed values of $\left(h_{i j}, \mathbf{P}^{i j}, \overline{\boldsymbol{\epsilon}}, \bar{P}, \widetilde{\boldsymbol{\epsilon}}, \tilde{P}, t, P_{t}, \bar{\theta}^{\alpha}, \overline{\mathbf{P}}_{\alpha}, \tilde{\theta}^{\alpha}, \tilde{\mathbf{P}}_{\alpha}\right)$ span the phase space of Hamiltonian general relativity on the manifold $M$. The canonical symplectic structure ${ }^{9}$ on this phase space then takes the form:

$$
\begin{aligned}
\Omega\left(\delta_{1}, \delta_{2}\right)= & \int_{\Sigma}\left\{\left(\delta_{2} \mathbf{P}^{i j}\right)\left(\delta_{1} h_{i j}\right)-\left(\delta_{1} \mathbf{P}^{i j}\right)\left(\delta_{2} h_{i j}\right)\right\}+\left\{\left(\delta_{2} P_{t}\right)\left(\delta_{1} t\right)-\left(\delta_{1} P_{t}\right)\left(\delta_{2} t\right)\right\} \\
& +\int_{\mathcal{B}}\left\{\left(\delta_{2} \bar{P}\right)\left(\delta_{1} \overline{\boldsymbol{\epsilon}}\right)-\left(\delta_{1} \bar{P}\right)\left(\delta_{2} \overline{\boldsymbol{\epsilon}}\right)\right\}+\int_{S}\left\{\left(\delta_{2} \tilde{P}\right)\left(\delta_{1} \widetilde{\boldsymbol{\epsilon}}\right)-\left(\delta_{1} \tilde{P}\right)\left(\delta_{2} \widetilde{\boldsymbol{\epsilon}}\right)\right\} \\
& +\int_{\mathcal{B}}\left\{\left(\delta_{2} \overline{\mathbf{P}}_{\alpha}\right)\left(\delta_{1} \bar{\theta}^{\alpha}\right)-\left(\delta_{1} \overline{\mathbf{P}}_{\alpha}\right)\left(\delta_{2} \bar{\theta}^{\alpha}\right)\right\}+\int_{S}\left\{\left(\delta_{2} \tilde{\mathbf{P}}_{\alpha}\right)\left(\delta_{1} \tilde{\theta}^{\alpha}\right)-\left(\delta_{1} \tilde{\mathbf{P}}_{\alpha}\right)\left(\delta_{2} \tilde{\theta}^{\alpha}\right)\right\} .
\end{aligned}
$$

9 This is really the pre-symplectic structure since the existence of the Hamiltonian and diffeomorphism constraints means that we have not isolated the true degrees of freedom. The proper symplectic structure would restrict itself to the true degrees of freedom. That said, we will follow the standard practice and refer to this pre-symplectic structure as the symplectic structure. 
Enforcing boundary conditions will impose restrictions on the allowed variations on $S$ and $\mathcal{B}$. There, we use the same weak boundary conditions that we considered in the action calculation in Section IIID as well as imposing the appropriate diffeomorphism constraints. We now consider how the conditions translate into the canonical framework.

First, on the outer boundary $\mathcal{B}$, the three-metric $\gamma_{i j}$ is a combination of the lapse $\bar{N}$, shift $\bar{V}^{A}$ and two-metric $\bar{\gamma}_{A B}$. We fix all of these quantities at the boundary, which in turn imposes restrictions on the full four-dimensional evolution at the boundary. From (4.1) we note that in particular this is sufficient to fix the bulk lapse $N$ and shift $V^{i}$, up to the freedom encoded in $\eta$ (or equivalently $\bar{P}$ ).

Next, we consider the inner, horizon, boundary $S$. The geometry of the horizon is naturally described in terms of the two-metric $\tilde{q}_{A B}$, shift vector $\tilde{V}^{A}$, the quantities $\alpha$ and $C$ and other quantities constructed from the null vector fields $\ell$ and $n$. These can, of course, be related to the three-dimensional metric $h_{i j}$, the momentum $\mathbf{P}^{i j}$, the lapse and shift, and the quantity $\zeta$. We will not give all the relations here, but refer the interested reader to Appendix B. In this case the boundary conditions are already tailored to the two-surface $S$. They require that we fix the two-metric $\tilde{q}_{A B}$, the angular momentum one-form $\tilde{\omega}_{A}$ and the value of $\alpha C$ (which is equivalent to the earlier fixing of $\underline{\ell}$ ), as well as requiring that $\theta_{(\ell)}$ vanish.

In analogy to the way that we restricted the variation of these quantities in the action formulation, here the variation of "fixed" quantities is restricted so that $\delta$ respectively acts as:

$$
\delta_{X_{S}}=(\delta t) \frac{d}{d t}+\mathcal{L}_{\widetilde{\delta X}} \quad \text { and } \quad \delta_{X_{\mathcal{B}}}=(\delta t) \frac{d}{d t}+\mathcal{L} \overline{\delta X}
$$

on $S$ and $\mathcal{B}$. Here, $\delta t$ is a function of $t$ alone (which means that the variation preserves the foliation) and $\widetilde{\delta X} / \overline{\delta X} \in T \mathcal{B} / S$. Finally, note the difference between this decomposition of the variation and that given in (4.5). In the earlier version, we decomposed with respect to $\mathcal{V}$ rather than $T$. Thus, the two are related by: $x_{o}=\delta t$ and $\widetilde{\delta X}^{A}=\tilde{x}^{A}-(\delta t) \tilde{V}^{A}$.

\section{B. Hamiltonian evolution}

In this section, we will use Hamiltonian methods to obtain expressions for the energy and angular momentum of a horizon. In order to do this, we will be interested in evolutions of the form

$$
\delta_{\Lambda}=\lambda_{o} \frac{d}{d t}+\mathcal{L}_{\lambda}
$$

where $\lambda_{o}=\lambda_{o}(t)$ is a scalar function and $\lambda^{i} \in T \Sigma$ is a vector field defined over $\Sigma$. In addition, we require that $\lambda^{i}$ be tangent on the boundaries. That is

$$
\lambda^{i}=p_{A}{ }^{i} \tilde{\lambda}^{A} \text { on } S \text { and } \lambda^{i}=p_{A}{ }^{i} \bar{\lambda}^{A} \text { on } \mathcal{B}
$$

for some $\tilde{\lambda}^{A} \in T S$ and $\bar{\lambda}^{A} \in T \mathcal{B}$. For an evolution along $\Lambda$, we will show that there exists a Hamiltonian functional which generates the evolution. Specifically, this means that the symplectic structure (4.4) with $\delta_{1}=\delta_{\Lambda}$ can be manipulated into the form

$$
\Omega\left(\delta_{\Lambda}, \delta\right)=\delta K_{\Lambda}+\text { constraints }
$$


This class of evolutions includes both time evolutions and rotations of the system, so we can expect the Hamiltonian functional $K_{\Lambda}$ to provide information about both the energy and the angular momentum associated with the horizon $S$, subject to an appropriate choice of $\Lambda$.

In order to rewrite the symplectic structure in the form (4.8) we must evaluate the bulk term by making use of the evolution equations for the three-metric and extrinsic curvature derived from the Einstein equations. This is a long calculation and we will simply state the result; we refer the interested reader to Appendix $\mathrm{B}$ for more details. Before giving the result, it is useful to introduce a shorthand

$$
H_{\Lambda}=\int_{\Sigma}\left\{\lambda_{o}\left(N \mathcal{H}+V^{i} \mathcal{H}_{i}\right)+\lambda^{i} \mathcal{H}_{i}\right\}
$$

for the bulk part of the Hamiltonian, where $\mathcal{H}={ }^{(\Sigma)} \boldsymbol{\epsilon} \mathcal{H}$ and $\mathcal{H}_{i}={ }^{(\Sigma)} \mathcal{\epsilon H}_{i}$. As usual this is comprised of constraints and will therefore vanish on shell. Then, we can express the bulk contribution to the symplectic structure as

$$
\begin{aligned}
\int_{\Sigma} & \left\{\left(\delta \mathbf{P}^{i j}\right)\left(\delta_{\Lambda} h_{i j}\right)-\left(\delta_{\Lambda} \mathbf{P}^{i j}\right)\left(\delta h_{i j}\right)\right\} \\
= & \delta\left(H_{\Lambda}-\int_{\mathcal{B}}\left\{\frac{\lambda_{o}}{8 \pi G} \overline{\boldsymbol{\epsilon}} \bar{N} k_{\bar{n}}+\overline{\boldsymbol{\epsilon}}\left(\lambda_{o} \bar{V}^{A}+\lambda^{A}\right) \bar{j}_{A}\right\}+\frac{1}{8 \pi G} \int_{S} \tilde{\boldsymbol{\epsilon}} \tilde{\lambda}^{A} \tilde{\omega}_{A}\right) \\
& -\int_{\Sigma}\left\{\delta\left(\lambda_{o} N\right) \boldsymbol{H}+\delta\left(\lambda^{i}+\lambda_{o} V^{i}\right) \mathcal{H}_{i}\right\} \\
& +\int_{\mathcal{B}}\left\{\left(\delta_{\Lambda} \bar{P}\right)(\delta \overline{\boldsymbol{\epsilon}})-(\delta \bar{P})\left(\delta_{\Lambda} \overline{\boldsymbol{\epsilon}}\right)\right\}+\int_{S}\left\{\left(\delta_{\Lambda} \tilde{\boldsymbol{\epsilon}}\right)(\delta \tilde{P})-(\delta \widetilde{\boldsymbol{\epsilon}})\left(\delta_{\Lambda} \tilde{P}\right)\right\} \\
& +\left(\delta \lambda_{o}\right) \int_{\mathcal{B}}\left\{\frac{\bar{N}}{8 \pi G} \overline{\boldsymbol{\epsilon}} k_{\bar{n}}+\overline{\boldsymbol{\epsilon}} \bar{\jmath}_{A} \bar{V}^{A}\right\}+(\delta t) \int_{\mathcal{B}} \mathcal{L} \mathcal{L}_{\Lambda}\left\{\frac{\bar{N}}{8 \pi G} \overline{\boldsymbol{\epsilon}}_{\bar{n}}+\overline{\boldsymbol{\epsilon}} \bar{\jmath}_{A} \bar{V}^{A}\right\} \\
& +\int_{\mathcal{B}}\left\{\left(\delta \bar{\lambda}^{\alpha}\right) \overline{\boldsymbol{\epsilon}} \bar{\jmath}_{\alpha}+\left(\delta \bar{\theta}^{\alpha}\right) \delta_{\Lambda}\left(\overline{\boldsymbol{\epsilon}} \bar{j}_{\alpha}\right)\right\}-\frac{1}{8 \pi G} \int_{S}\left\{\left(\delta \tilde{\lambda}^{\alpha}\right) \widetilde{\boldsymbol{\epsilon}} \tilde{\omega}_{\alpha}+\left(\delta \tilde{\theta}^{\alpha}\right) \delta_{\Lambda}\left(\widetilde{\boldsymbol{\epsilon}} \tilde{\omega}_{\alpha}\right)\right\} .
\end{aligned}
$$

In order to obtain the above, we have made repeated use of the boundary conditions. Specifically, we have used $\theta_{(\ell)}=0$ and the fact that all other "fixed" quantities vary as described in (4.5). Additionally, we have made use of the diffeomorphism constraints on both the horizon and outer boundary. Then, (4.10) is close to the form that we require. The first term is an exact variation, and will be a contribution to the Hamiltonian $K_{\Lambda}$, while the second line will vanish provided the bulk constraints are satisfied. The third line consists of terms involving the boundary area forms and their conjugate momenta. The final two lines involve additional terms on the boundaries. These terms will eventually lead to our expressions for the energy and angular momentum of the horizon and outer boundary.

To shorten and clarify our expressions we introduce further notation. First, we define

$$
\begin{aligned}
K_{t} & :=P_{t}-\int_{\mathcal{B}} \overline{\boldsymbol{\epsilon}}\left\{\frac{1}{8 \pi G} \bar{N} k_{\bar{n}}+\bar{V}^{A} \bar{\jmath}_{A}\right\} \\
\overline{\boldsymbol{L}}_{A}: & :=\overline{\mathbf{P}}_{A}-\overline{\boldsymbol{\epsilon}} \bar{\jmath}_{A} \text { and } \tilde{\boldsymbol{L}}_{A}:=\tilde{\mathbf{P}}_{A}+\frac{\tilde{\boldsymbol{\epsilon}}}{8 \pi G} \tilde{\omega}_{A} .
\end{aligned}
$$


We then substitute (4.10) into the symplectic structure (4.4). Then,

$$
\begin{aligned}
\Omega\left(\delta_{\Lambda}, \delta\right)= & \delta\left(H_{\Lambda}+\lambda_{o} K_{t}+\int_{\mathcal{B}} \bar{\lambda}^{A} \overline{\boldsymbol{L}}_{A}+\int_{S} \tilde{\lambda}^{A} \tilde{\boldsymbol{L}}_{A}\right) \\
& -\int_{\Sigma} d^{3} x\left\{\delta\left(\lambda_{o} N\right) \mathcal{H}+\delta\left(\lambda_{o} V^{i}+\lambda^{i}\right) \boldsymbol{\mathcal { H }}_{i}\right\} \\
& -\left(\delta \lambda_{o}\right) K_{t}-\int_{\mathcal{B}}\left(\delta \bar{\lambda}^{\alpha}\right) \overline{\boldsymbol{L}}_{\alpha}-\int_{S}\left(\delta \tilde{\lambda}^{\alpha}\right) \tilde{\boldsymbol{L}}_{\alpha} \\
& -(\delta t)\left(\delta_{\Lambda} K_{t}\right)-\int_{\mathcal{B}}\left(\delta \bar{\theta}^{\alpha}\right)\left(\delta_{\Lambda} \overline{\boldsymbol{L}}_{\alpha}\right)-\int_{S}\left(\delta \tilde{\theta}^{\alpha}\right)\left(\delta_{\Lambda} \tilde{\boldsymbol{L}}_{\alpha}\right) .
\end{aligned}
$$

The first term line in (4.12) is an exact variation. Therefore, if we can argue that the remaining terms vanish, this term will be the Hamiltonina $K_{\Lambda}$ which generates evolutions along $\Lambda$. This will be the case provided the five quantities $\mathcal{H}, \mathcal{H}_{i}, K_{t}, \overline{\boldsymbol{L}}_{\alpha}$ and $\tilde{\boldsymbol{L}}_{\alpha}$ are all zero. In addition, the evolution along $\Lambda$ of $K_{t}, \overline{\boldsymbol{L}}_{\alpha}$ and $\tilde{\boldsymbol{L}}_{\alpha}$ must also vanish.

The vanishing of $\mathcal{H}$ and $\mathcal{H}_{i}$ is nothing more than the usual Hamiltonian and diffeomorphism constraints. The other conditions restrict the values of the momenta $P_{t}$ and $\mathbf{P}_{\alpha}$. Until this point, these quantities have been entirely free, but now we must require

$$
\begin{aligned}
P_{t} & =\int_{\mathcal{B}} \overline{\boldsymbol{\epsilon}}\left\{\frac{\bar{N} k_{\bar{n}}}{8 \pi G}+\bar{V}^{A} \bar{\jmath}_{A}\right\}, \\
\overline{\mathbf{P}}_{\alpha} & =\overline{\boldsymbol{\epsilon}} \bar{\jmath}_{\alpha} \text { and } \tilde{\mathbf{P}}_{\alpha}=-\frac{\widetilde{\boldsymbol{\epsilon}}}{8 \pi G} \tilde{\omega}_{\alpha} .
\end{aligned}
$$

Next, the fact that the $\Lambda$ variation of $K_{t}$ and $\boldsymbol{L}_{\alpha}$ vanishes fixes the evolution of the momenta. Specifically, it guarantees that the functional forms given in (4.13) and (4.14) are preserved in time. Thus, our evolution will be generated by a Hamiltonian provided that the standard bulk constraints of general relativity are satisfied and in addition the conjugate momenta introduced on the boundaries take the form given above in (4.13) and (4.14).

Thus, if these equations of motion are imposed in the extended phase space, $\delta_{\Lambda}$ evolutions are generated by the Hamiltonian

$$
K_{\Lambda}=H_{\Lambda}+\lambda_{o} K_{t}+\int_{\mathcal{B}} \overline{\boldsymbol{\epsilon}} \bar{\lambda}^{A} \overline{\boldsymbol{L}}_{A}+\int_{S} \tilde{\boldsymbol{\epsilon}} \tilde{\lambda}^{A} \tilde{\boldsymbol{L}}_{A}
$$

As always for a Hamiltonian, this is constant on-shell. In fact it vanishes since it is composed entirely of constraints. However, in the extended phase space, this does not mean that the energy and angular momenta are identically zero. Instead, the energy is given by the value of the momentum canonically conjugate to the time variable. Thus, the formalism tells us that the energy associated with the evolution $\frac{d}{d t}$ is

$$
E[d / d t]=\int_{\mathcal{B}} \bar{\epsilon}\left\{\frac{\bar{N} k_{\bar{n}}}{8 \pi G}+\bar{V}^{A} \bar{\jmath}_{A}\right\} .
$$

The value of energy we have obtained from the phase space calculation agrees with the one arising from the Legendre transform in the previous section. Again, we see that there is no horizon contribution. Similarly, the angular momenta of the boundaries are determined by 
the momenta $\mathbf{P}_{\alpha}$. Therefore, the angular momentum $J[\phi]$ associated with a vector field $\phi^{A}$ at the two boundaries is

$$
J_{\mathcal{B}}[\phi]=\int_{\mathcal{B}} \overline{\boldsymbol{\epsilon}} \phi^{A} \bar{\jmath}_{A} \quad \text { and } \quad J_{S}[\phi]=-\frac{1}{8 \pi G} \int_{S} \tilde{\boldsymbol{\epsilon}} \phi^{A} \tilde{\omega}_{A}
$$

We thus obtain angular momentum values for both boundaries independently. The value at the outer boundary agrees with what is generally obtained. The expression for the angular momentum of the horizon provides an extension of the previous definition (2.6) on an isolated horizon. This phase space calculation tells us that we can associate an angular momentum with any vector field $\phi$ at the horizon, not just axisymmetric Killing vectors. As expected, the value of the angular momentum is determined by the field $\tilde{\omega}_{A}$.

\section{Freedom in the Hamiltonian}

Just as there was a freedom in the definition of the action $I_{H}$ and the quasilocal energy $H(t)$, there is a corresponding freedom in the definition of our Hamiltonian $K_{\Lambda}$. A close examination of (4.12) reveals that $K_{\Lambda}$ is not the only Hamiltonian that generates the evolution $\delta_{\Lambda}$ through the physical phase space. Consider for example

$$
K_{\Lambda}^{\prime}=K_{\Lambda}+\lambda_{o} F(t)
$$

where $F(t)$ is a free function. Then, it is fairly easy to see that this functional also generates the usual physical evolutions subject to a modification of the constraint equation (4.13) so that it becomes

$$
P_{t}=\int_{\mathcal{B}} \overline{\boldsymbol{\epsilon}}\left\{\frac{\bar{N} k_{\bar{n}}}{8 \pi G}+\bar{V}^{A} \bar{\jmath}_{A}\right\}+F(t) .
$$

In the extended phase space, this corresponds to a displacement to an alternate constraint surface that still corresponds to the usual physical evolution of the metric and extrinsic curvature. In fact one can argue that this is the most general term that can be added to the Hamiltonian [15]. With this alternate Hamiltonian, the quasilocal energy becomes

$$
E[d / d t]=F(t)+\int_{\mathcal{B}} \bar{\epsilon}\left\{\frac{\bar{N} k_{\bar{n}}}{8 \pi G}+\bar{V}^{A} \bar{\jmath}_{A}\right\} .
$$

The quasilocal angular momentum $J_{\phi}$ remains unchanged.

Mathematically, $F(t)$ can take any form but from a physical point of view the only functionals that make sense are those constructed from from the time dependent boundary data. That is

$$
F(t)=F\left[\bar{\gamma}_{A B}, \bar{N}, \bar{V} ; \tilde{q}_{A B}, \tilde{\omega}_{A}, \alpha C, \theta_{(\ell)}\right]
$$

and so the quasilocal energy derived by this method is compatible with that (3.38) found by action arguments. 


\section{ENERGY AND ANGULAR MOMENTUM OF THE HORIZON}

In Sections III and IV we have obtained expressions for the energy and angular momentum of a spacetime containing a horizon as a boundary. In this section we will consider these expressions in more detail. In particular we will show that the expression for angular momentum is equivalent to the corresponding expressions on isolated and dynamical horizons. Turning to the energy we will see that although a wide range of energy expressions are allowed by our mathematical formalism, this freedom may be greatly reduced through the imposition of a few natural conditions. Once this is done, the remaining freedom is essentially that found in the dynamical horizon formalism.

\section{A. Angular Momentum}

The angular momentum of the horizon, derived using the Hamiltonian framework, is given by

$$
J_{S}[\phi]=-\frac{1}{8 \pi G} \int_{S} \tilde{\boldsymbol{\epsilon}} \phi^{A} \tilde{\omega}_{A} .
$$

This expression is unique, as the freedom we have to add terms to the Hamiltonian $K_{\Lambda}$ will not affect the value of the angular momentum obtained. Furthermore, this value of angular momentum is applicable for both isolated and dynamical horizons. It is immediate from (2.6) that our expression is identical to the standard one on an isolated horizon.

The dynamical horizon definition for angular momentum (2.7) is

$$
J_{D H}[\phi]=\frac{1}{8 \pi G} \int_{S} \tilde{\boldsymbol{\epsilon}} \phi^{a} \hat{r}^{b} \nabla_{a} \hat{\tau}_{b} .
$$

This is clearly very similar to the expression that we derived by Hamiltonian methods, but it isn't identical - (5.1) made use of the null normals $\ell^{a}$ and $n^{a}$ whereas (5.2) uses the spacelike normals $\hat{r}^{a}$ and $\hat{\tau}^{a}$. We will now show that for reasonable choices of $\phi^{A}$, this difference is irrelevant and either choice of normals will give the same value for the angular momentum (up to an overall sign) associated with $\phi^{A}$ on the horizon.

To see this, let us consider the conditions that $\phi^{A}$ should meet so that it may be viewed as a generator of rotations. We start with the example of a two-dimensional slice of the Kerr horizon. There, the obvious choice for $\phi^{A}$ is the Killing vector field, $(\partial / \partial \phi)$ in the usual coordinate systems. Note that in addition to satisfying $\mathcal{L}_{(\partial / \partial \phi)} \tilde{q}_{A B}=0$, this vector field can also be integrated to foliate the two-slice into a family of closed curves, all of which have a common period.

With this example in mind we will assert that $\phi^{A}$ is a suitable "rotation" vector field if :

1. It integrates to a congruence of closed curves that foliate $S$ (rotations should be periodic!).

2. $\mathcal{L}_{\phi} \widetilde{\boldsymbol{\epsilon}}=0 \Leftrightarrow d_{A} \phi^{A}=0$. Given the first condition, this one may always be met through a rescaling of $\phi^{A}$. Specifically, $\phi^{A}$ is restricted to take the form

$$
\phi^{A}=\beta \tilde{\epsilon}^{A B} d_{B} \theta
$$

where $\theta$ is a labelling of the congruence and $\beta=\beta(\theta)$ is a free function. This is a necessary condition for $\phi^{A}$ to be a Killing vector field. It may be thought of a requiring a kind of uniformity of $\phi^{A}$ along each curve. 
3. Finally, $\beta$ should be chosen so that the curves all integrate to have a common period (rotations should have a common period for all orbits!).

A $\phi^{A}$ of this type satisfies many, but obviously not all, of the properties of a Killing vector that generates rotational symmetries. It is not hard to show that these conditions are more than sufficient to force the two angular momentum expressions to be equivalent. Begin by noting that on a (spacelike) dynamical horizon,

$$
\ell^{a}=\sqrt{\frac{C}{2}}\left(\hat{\tau}^{a}+\hat{r}^{a}\right) \text { and } n^{a}=\frac{1}{\sqrt{2 C}}\left(\hat{\tau}^{a}-\hat{r}^{a}\right) .
$$

Whence, it easily follows that

$$
\begin{aligned}
\int_{S} \tilde{\boldsymbol{\epsilon}} \phi^{A} \tilde{\omega}_{A} & =\int_{S} \tilde{\boldsymbol{\epsilon}}\left\{\phi^{a} \hat{r}_{b} \nabla_{a} \hat{\tau}^{b}+\frac{1}{2} \phi^{A} d_{A}(\ln C)\right\} \\
& =\int_{S} \widetilde{\boldsymbol{\epsilon}}\left\{\phi^{a} \hat{r}_{b} \nabla_{a} \hat{\tau}^{b}+\frac{1}{2} d_{A}\left(\phi^{A} \ln C\right)-\frac{1}{2} \ln C d_{A} \phi^{A}\right\} .
\end{aligned}
$$

The second term on the second line vanishes because $S$ is closed and the third term vanishes since $\phi^{A}$ is required to be divergence-free.

Thus, with $\phi^{A}$ of the class that we have discussed, the angular momentum expression that we derived by Hamiltonian arguments (5.1) agrees exactly with the expression that appears in the Ashtekar-Krishnan flux law (5.2). The two calculations then support each other. Our derivation gives a Hamiltonian justification for viewing this expression as representing angular momentum, while the flux law then shows how to calculate changes in that angular momentum due to matter and energy crossing the horizon.

There are a couple of points to keep in mind with respect to these expressions. First, the derivation of the dynamical horizon flux law does not actually make use of the fact that $H$ is a dynamical horizon, except for assuming it is spacelike (so that $\hat{r}^{a}$ and $\hat{\tau}^{a}$ are defined). Thus, it is true for all spacelike surfaces, not just horizons. Second, although [9] is the first paper to derive a flux law of this type for spacelike surfaces, very similar angular momentum definitions and flux laws have appeared for timelike and null surfaces. In fact, while expressions for quasilocal energy vary widely, angular momentum is almost always defined with respect to the connection on the normal bundle to a two-surface. Examples of these expressions include: the Komar formula for stationary spacetimes (see, for example, [26]), the definition of angular momentum for an isolated horizon [8], and the Brown-York definition for quasilocal angular momentum [11]. The only difference between the various definitions is which normal vectors to $S$ are used in the angular momentum expression.

\section{B. Energy}

Both the action and Hamiltonian calculations allow for the horizon energy to be any free functional of the fixed data. That is,

$$
\text { Energy }=E\left[\tilde{q}_{A B}, \tilde{\omega}_{A}, \theta_{(\ell)}, \underset{\leftarrow}{\ell}, v\right],
$$

where the exact form of $E$ is unspecified. However, it is also clear that many of these functionals will not be physically acceptable. To narrow down the choices, we impose the following conditions on the energy functional: 
1. Dimensional correctness: When $c=G=1, E$ should have units of length.

2. Constancy in isolation: E should be constant over isolated sections of horizon.

3. Scaling with time: E should rescale linearly with reparameterizations of time.

All three of these are very natural. In particular any functional that doesn't satisfy the first two conditions could hardly be considered an energy. The third is a little less precise, but is also very fundamental. Energy is conjugate to time and so its value should change with rescalings of time. However, what has been left imprecise in this statement is exactly what we mean by time. We will now consider this point.

On the outer boundary $B$, the evolution vector field $T^{a}$ is naturally identified with the flow of time. There, $T^{a}$ appears in the energy functional

$$
E_{\mathcal{B}}=\int_{\mathcal{B}} \tilde{\boldsymbol{\epsilon}}\left(\frac{\bar{N} k_{\bar{u}}}{8 \pi}+\bar{V}^{A} \bar{\jmath}_{A}\right)=\int_{\mathcal{B}} \widetilde{\boldsymbol{\epsilon}} T^{a}\left(\frac{-k_{\bar{u}} \bar{u}_{a}}{8 \pi}+\bar{\jmath}_{a}\right) .
$$

Clearly this is dimensionally correct and also rescales with $T^{a}$. Further, since $T^{a}$ is fixed data, any extra reference terms that are added may also be given the correct scaling behaviour. Given this experience, on $H$ the obvious first candidate to consider as a time vector is $(\partial / \partial v)$ or possibly $\mathcal{V}$. However, with the horizon boundary conditions, this cannot be the correct choice as these vectors neither appear in our base expression (which is 0 on $H$ ) nor in the fixed data. The only way that we could get a rescaling with respect to these would be through the foliation parameter $v$. However, an expression of this type would clearly fall afoul of condition 2 and not be constant on isolated horizons.

Thus, the energy associated with the surface $S$ cannot rescale with either $(\partial / \partial v)$ or $\mathcal{V}$. However, among the fixed data we do have $\underset{\leftarrow}{\leftarrow}=\alpha C d v$. We are then naturally lead to consider an energy functional that has a linear dependence on $\underline{\ell}$. Furthermore, the null vector field $\ell^{a}$ is causal which gives it an additional attraction over other, possibly acausal, alternatives. In considering possible forms of the energy functional, we note that over isolated regions where $\ell_{a}$ is normal to $H, \underline{\ell}=0$. Therefore, we propose an energy functional of the following form:

$$
d E=\frac{1}{c_{o} a} \int_{S} \stackrel{\ell}{\leftarrow} \wedge \widetilde{\boldsymbol{\epsilon}}
$$

where $a$ is the area of $S$ and $c_{o}$ is a constant. That is, we argue that the energy flux should be proportional to the "average" value of $\underline{\ell} .{ }^{10}$ Then, given cross-sections $S_{1}$ and $S_{2}$ which bound a region $\Delta H$ their energies are related by:

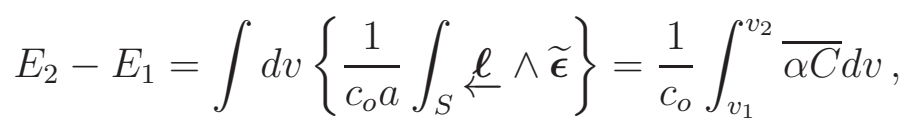

where $\overline{\alpha C}=\frac{1}{a} \int_{S} \tilde{\boldsymbol{\epsilon}} \alpha C$ is the average value of $\alpha C$ over any surface. Thus, for any choice of $\ell$, an energy defined in this way will meet our minimum requirements: it will be dimensionally correct, constant when $H$ is isolated, and (its flux) will rescale with respect to a causal

\footnotetext{
10 Note that our freedoms would equally well allow us to multiply the right-hand side of this equation by any dimensionless functional $f\left[\tilde{q}_{A B}, \tilde{\omega}_{A}, v\right]$ of the remaining fixed data. However, since $\ell$ may already be freely chosen, this does not add to the generality of the expression.
} 
"time" vector field. However, the energy in (5.9) does not fit naturally into the allowed freedoms of the action formulation - we would have to define $E$ as an integral of the fixed data from some reference surface $S_{o}$ with energy $E_{o}$ to the surface $S-$ and is even less natural in the Hamiltonian framework. Therefore, we will further restrict the freedom in $\underline{\ell}$, and hence the form of the energy.

For a specific horizon geometry, we would like to fix a unique normalization for $\ell$ and consequently a preferred value for the energy. This is familiar from, for example, the Kerr isolated horizon. There we obtain a family of permissible evolution vector fields $\ell$, but restrict to a preferred one by requiring it to have a specific value of surface gravity. To make a similar fix here, we need to tie $\underline{\ell}$ to the geometry of the horizon. If the horizon does not contain any "partially-isolated" two-surfaces ${ }^{11}$ then a nice way to do this is to require that $\stackrel{\ell}{\longleftarrow}$ satisfy

$$
\underline{\ell}=c_{o} d f\left[\tilde{q}_{A B}, \tilde{\omega}_{A}\right] \quad \Leftrightarrow \quad \alpha C=c_{o}\left(\frac{d f}{d v}\right),
$$

where $f=f\left[\tilde{q}_{A B}, \tilde{\omega}_{A}\right]$ is some functional of the fixed geometric data on each two-surface $S$. Recently it has been shown that a wide class of $\theta_{(\ell)}=0$ three-surfaces actually meet this assumption [35]. Roughly speaking, if there are trapped surfaces arbitrarily close to (though spacelike-separated from) each two-surface then on those $\left(\theta_{(\ell)}=0\right)$ surfaces $\ell$ vanishes either everywhere or nowhere. Thus, for most $H$ which can be identified as black hole boundaries, one should be able to fix $\underline{\ell}$ in this way.

With such a fix, $\ell \mathfrak{l}$ is "constant" over each leaf of the foliation. Then, consulting (5.8) it is not hard to see that this will uniquely determine the flux of energy through the horizon between cross-sections - and so the actual energy expression up to the usual additive constant. However, in general relativity the only dimensionful constants are the speed of light $c$ and Newton's constant $G$. From these, it is impossible to construct a quantity with units of energy whence it is natural to set the additive constant to zero. Then, we obtain

$$
E=f\left[\tilde{q}_{A B}, \tilde{\omega}_{A}\right]
$$

Thus, with this choice the energy is fixed directly in terms of the geometry and $\underset{\ell}{\ell}$ is fixed up to an overall constant rescaling.

The energy expression derived above has another interesting property — it must always be non-decreasing. This follows immediately from the fact that the pull-back of the forwardin-time pointing $\ell$ will always be positive on a dynamical horizon $H$ (or zero in isolation). Then, with Kerr black holes and the Penrose process in mind, this suggests that we associate $E$ with the irreducible mass of the horizon (see for example [16]) rather than the "total" mass which can either increase or decrease in time. That is we define

$$
f=\frac{R}{2}
$$

where $R$ is the areal radius defined by $a=: 4 \pi R^{2}$.

If one wished to instead consider the "total" energy it would be necessary to consider a more complicated evolution vector field. Specifically in the case of axisymmetry, it would be natural to consider $\ell+\Omega \phi$, where $\phi$ is the rotational Killing vector field and $\Omega$ is a

${ }^{11}$ That is, two-surfaces which contain both regions where $\underline{\ell}=0$ and others where $\underline{\ell} \neq 0$. 
function of the fixed data. Then the "irreducible" energy would remain unchanged since $\ell+\Omega \phi \wedge \widetilde{\boldsymbol{\epsilon}}=\stackrel{\ell}{\llcorner} \wedge \widetilde{\boldsymbol{\epsilon}}$. However, $\phi^{A}$ would enter the fixed data as a symmetry of the two-metric, and so one could also include it in energy expressions. In particular, with this symmetry there would be an unambiguous angular momentum $J$ for each cross-section of the horizon and this could be used to construct energy expressions like the usual Smarr formula $E(a, J)$ for the total mass of a Kerr black hole.

\section{Relation to dynamical horizons}

We will now show that the energy obtained by Hamiltonian methods is related to the dynamical horizon energy and flux laws. Furthermore, by appealing to dynamical horizons, we can fix the unknown constant $c_{o}$ and hence further restrict the energy. We begin by reviewing those aspects of the dynamical horizon calculation which are relevant here.

Dynamical horizons are required to be spacelike, therefore, we can introduce the unit timelike and spacelike normals $\hat{\tau}^{a}$ and $\hat{r}^{a}$. From these, we obtain a null vector

$$
\hat{\ell}^{a}=\hat{\tau}^{a}+\hat{r}^{a} .
$$

The dynamical horizon flux law is obtained by integrating a combination of the Hamiltonian and diffeomorphism constraints over the horizon. The specific combination is chosen to correspond to the $\hat{\ell}^{a} \hat{\tau}^{b}$ component of the Einstein equations. Then, by making use of the fact that at the horizon $\theta_{(\ell)}=0$, one obtains an energy flux law

$$
E_{2}-E_{1}=\int_{H}{ }^{(H)} \boldsymbol{\epsilon} T_{a b} \xi_{E}^{a} \hat{\tau}^{b}+\frac{1}{16 \pi G} \int_{H}{ }^{(H)} \boldsymbol{\epsilon} N_{E}\left\{\sigma_{(\ell)}^{A B} \sigma_{(\ell) A B}+2 \zeta^{A} \zeta_{A}\right\}
$$

In the above, ${ }^{(H)} \boldsymbol{\epsilon}$ is the horizon volume form, $\zeta_{A}=p_{A}^{a} \hat{r}^{b} \nabla_{b} \hat{\ell}_{a}, N_{E}$ is the lapse on the horizon and $\xi_{E}^{a}$ is the evolution vector field associated with the energy functional $E$. Finally, the energy and evolution vector are tied together via the lapse by

$$
N_{E}=2\|d E\| \quad \text { and } \quad \xi_{E}^{a}=N_{E} \hat{\ell}^{a} .
$$

The expression (5.14) provides a nice relationship between the change in energy of a horizon and the flux of gravitational and matter energy through the horizon. The change in energy is shown to be equal to three terms, all of which are non-negative (provided the weak energy condition is satisfied). The first is identified as the flux of matter through the horizon and the second is the flux of gravitational shear. There is no clear interpretation of the third contribution.

Thus, the dynamical horizon energy always increases in the same way as the "irreducible" energy that we have derived. Further, we note that (5.15) can be rewritten without reference to unit vectors (with the help of (5.13) ) as

$$
\xi=2 d E .
$$

This suggests that we fix the unknown constant arising in (5.10) as $c_{o}=1 / 2$. Then, the Hamiltonian evolution vector field $\ell^{a}$ is equal to the evolution vector $\xi^{a}$ arising in the flux law (5.14). Therefore, the Hamiltonian evolution vector field and energy also satisfy the dynamical horizon flux law. 
In summary, a simple argument has lead us to obtain an energy functional for the horizon. This energy is constant whenever the horizon is isolated. Additionally, by arguing that the energy should rescale appropriately with reparametrizations of time, we have identified the null vector $\ell$ as the evolution vector field of the horizon. This is a surprising result. One would usually expect the evolution vector field to be tangent to the boundary, but in this case it will not be, unless the horizon is isolated. Next, by relating the value of $\ell$ to the other geometrical properties of the horizon, we obtain a energy associated to $\ell$ which is unique up to an overall constant rescaling. It is non-decreasing and so naturally associated with the irreducible (rather than total) mass of the horizon. Finally, by appealing to the dynamical horizon results (which also describe non-decreasing energies), we can restrict the little remaining freedom in the energy in such a way that $\ell$ and $E_{\ell}$ satisfy the dynamical horizon flux laws.

\section{DISCUSSION}

There are many different local definitions for black hole horizons: apparent, trapping, dynamical, isolated, and slowly evolving. Although not identical, these all share some common features. Specifically, they are all three-surfaces which can be foliated by two-surfaces of zero outward expansion. More precisely, for each two-surface the outward directed null normal $\ell$ to the surface has zero expansion, $\theta_{(\ell)}=0$. In this paper, we presented both action and canonical phase space formulations for a spacetime with such a boundary. These formulations provide us with quasi-local expressions for the horizon energy and angular momentum.

A trapping horizon has one natural boundary condition, namely that the outward expansion $\theta_{(\ell)}$ vanishes. This in itself is a mixed boundary condition in the sense that it depends upon both the intrinsic and extrinsic geometry of the surface. We impose additional boundary conditions which fix the two-metric $\tilde{q}_{A B}$ and the one-form $\tilde{\omega}_{A}$ on every slice of the horizon. The one-form $\tilde{\omega}_{A}$ is the connection on the normal bundle to the horizon. Physically, these fix the intrinsic geometry of the two-surfaces as well as their associated angular momenta. Unlike the $\theta_{(\ell)}=0$ condition which holds for all rescalings of the null vector $\ell$, the one-form $\tilde{\omega}_{A}$ depends upon the particular normalization of $\ell$. Thus, we must also fix a preferred normalization for $\ell$, and this is done by fixing the pull-back of $\ell$ to the horizon.

We have shown that these are suitable boundary conditions for both the action and Hamiltonian formulations of general relativity. While this is an interesting result in its own right, in this paper we are mostly interested in the expressions that we can then derive for the energy and angular momentum associated with the horizon two-surfaces. The result for the angular momentum is unambiguous: there is a definite value of angular momentum associated with each cross section of the horizon. Furthermore, this value is identical to the angular momentum calculated for axisymmetric isolated horizons. In addition, if we restrict attention to divergence free vector fields, the value of the angular momentum agrees with that arising in the dynamical horizon flux law.

Obtaining an energy for the horizon is not so straightforward. As in all quasilocal Hamiltonian calculations, there is a freedom to add a functional of the fixed boundary data to the Hamiltonian and hence change the value of the energy. However, we can almost entirely remove this freedom by requiring that the energy satisfy a few simple physical properties. These then lead to several interesting results. The first is that the appropriate "evolution 
vector" for the horizon is the null vector $\ell$ and hence, in contrast to a typical Hamiltonian formulation, the evolution vector need not map cross sections of the horizon into each other. Next, by requiring that the null vector $\ell$, or more accurately its pull-back to the horizon, is compatible with the horizon geometry we obtain a range of possible expressions for the energy of the horizon: For a particular choice of null vector field $\ell$ the horizon energy is fixed up to an overall constant rescaling. All of these energies are constant on isolated horizons and increasing on dynamical horizons, and can therefore be interpreted as describing the irreducible mass of the horizon. Finally, appealing to the dynamical horizon formulation we can then fix the value of the rescaling constant relating the energy to the evolution vector $\ell$. Once this is done, we find that our energy $E_{\ell}$ and evolution vector field $\ell$ satisfy the dynamical horizon flux law.

Note however that this is certainly not meant to imply that all problems concerning energy and dynamical horizons have been resolved. While it is remarkable that the allowed energy expressions obtained by these two very different methods agree, it is also important to keep in mind that neither method singles out a unique expression. While this is somewhat disappointing it is also not too surprising given the well-known ambiguities of gravitational energy.

Other problems also remain for future investigations. Probably the most obvious arises on recalling the discussion of footnote 2. There it was pointed out that these $\theta_{(\ell)}=0$ horizons are not uniquely defined - in most spacetimes a given horizon will be part of an entire family of $\theta_{(\ell)}=0$ three-surfaces which may be smoothly deformed into each other. Thus, in using these techniques to discuss energy flows, one needs to be careful as quantities such as cross-sectional area will certainly depend on the member of the family chosen. This is not necessarily a problem. In physics, it is common for observations to vary with reference frames (think special relativity). The important thing is that invariant physical predictions should be compatible. Thus, for example, while the total energy absorbed by a black hole during a finite incidence of gravitational waves should be invariant, the details of how that happens may depend on the specific "horizon".

Lastly, the formalism and boundary conditions presented here may find other applications. Perhaps the most interesting is the possibility that the boundary conditions that we have discussed could find a use in quantum gravity discussions of dynamic black holes - just as the isolated horizon boundary conditions and Hamiltonian formalism found an application in the loop quantum gravity computations of black hole entropy.

\section{Acknowledgements}

We would like to thank Abhay Ashtekar, Christopher Beetle, Jolien Creighton and Badri

Krishnan for useful discussions and also acknowledge the careful reading and constructive suggestions of our referees. S.F. was supported by the Killam Trusts at the University of Alberta and NSF Grant No PHY-0200852; I.B. was supported by NSERC. 


\section{APPENDIX A: DETAILS OF THE ACTION VARIATION}

In this section we show that

$$
\begin{aligned}
& \delta I_{H}=\frac{1}{16 \pi G} \int_{M} \boldsymbol{\epsilon} G_{a b} \delta g^{a b}-\frac{1}{16 \pi G} \int_{\Sigma_{2}-\Sigma_{1}}^{(\Sigma)}\left(K_{\hat{u}}^{i j}-K_{\hat{u}} h^{i j}\right) \delta h_{i j} \\
& +\frac{1}{16 \pi G} \int_{B}^{(B)} \boldsymbol{\epsilon}\left(K_{\bar{n}}^{i j}-K_{\bar{n}} \gamma^{i j}\right) \delta \gamma_{i j}+\frac{1}{8 \pi G} \int_{\mathcal{B}_{2}-\mathcal{B}_{1}}(\delta \overline{\boldsymbol{\epsilon}}) \sinh ^{-1}(\eta) \\
& +\frac{1}{16 \pi G} \int_{H} \mathbf{d} \boldsymbol{v} \wedge \widetilde{\boldsymbol{\epsilon}}\left\{\tilde{s}^{A B} \delta \tilde{q}_{A B}\right\}+\frac{1}{8 \pi G} \int_{S_{2}-S_{1}}(\delta \widetilde{\boldsymbol{\epsilon}})(1-\ln \zeta) \\
& +\frac{1}{8 \pi G} \int_{H}\left\{\tilde{\boldsymbol{\epsilon}} \wedge\left((\delta \underline{\ell}) \theta_{(n)}-(\delta \underline{\boldsymbol{n}}) \theta_{(\ell)}\right)+\tilde{V}^{A} \delta\left(\mathbf{d} \boldsymbol{v} \wedge \tilde{\boldsymbol{\epsilon}} \tilde{\omega}_{A}\right)-\delta\left(2 \underline{\boldsymbol{n}} \theta_{(\ell)} \wedge \tilde{\boldsymbol{\epsilon}}\right)\right\} \text {. }
\end{aligned}
$$

as asserted at equation (3.10). In the interests of brevity, the (well-known) outer boundary terms on $B$ will be omitted from this derivation. Details of the calculations for those terms can be found in a standard reference such as [16, 26].

We begin by writing the bulk term in the action as:

$$
\delta(\boldsymbol{\epsilon} \mathcal{R})=\boldsymbol{\epsilon}\left\{G_{a b} \delta g^{a b}+\nabla^{a}\left[g^{b c}\left(\nabla_{b} \delta g_{a c}-\nabla_{a} \delta g_{b c}\right)\right]\right\}
$$

On integrating over $M$ and using Stokes theorem to move total derivatives out to the boundaries this becomes:

$$
\begin{aligned}
\delta\left(\int_{M} \boldsymbol{\epsilon} \mathcal{R}\right)= & \int_{M} \boldsymbol{\epsilon} G_{a b} \delta g^{a b}+\int_{\Sigma_{2}-\Sigma_{1}}^{(\Sigma)} \hat{u}^{a} g^{b c}\left(\nabla_{b} \delta g_{a c}-\nabla_{a} \delta g_{b c}\right) \\
& +\int_{H} \mathbf{d} \boldsymbol{v} \wedge \widetilde{\boldsymbol{\epsilon}} \tau^{a} g^{b c}\left(\nabla_{b} \delta g_{a c}-\nabla_{a} \delta g_{b c}\right) .
\end{aligned}
$$

In order to obtain the term on the horizon, we have used the expression (3.6) for the volume form. With just a little algebra, the term on $\Sigma_{1,2}$ can be written as:

$$
\begin{aligned}
& \int_{\Sigma_{2}-\Sigma_{1}}^{(\Sigma)} \boldsymbol{\epsilon} \hat{u}^{a} g^{b c}\left(\nabla_{b} \delta g_{a c}-\nabla_{a} \delta g_{b c}\right) \\
& \quad=-\int_{\Sigma_{2}}\left\{\begin{array}{c}
{ }_{\Sigma_{1}}^{(\Sigma)} \boldsymbol{\epsilon} \\
\boldsymbol{\epsilon}
\end{array}\left(K_{\hat{u}}^{i j}-K_{\hat{u}} h^{i j}\right) \delta h_{i j}+2 \delta\left({ }^{(\Sigma)} \boldsymbol{\epsilon} K_{\hat{u}}\right)\right\}-\int_{S_{2}-S_{1}} \tilde{\boldsymbol{\epsilon}} \hat{n}_{a} \delta \hat{u}^{a} .
\end{aligned}
$$

The last term is not, of course, zero since variations of the "time-space" components of $g^{a b}$ can give $\delta \hat{u}^{a}$ a component in the $\hat{n}_{a}$ direction. This is in contrast to $\delta \hat{u}_{a}$ whose "direction", though not normalization, is fixed by the requirement that it be normal to the $\Sigma_{1,2}$ surfaces.

The signature on $H$ is indeterminate, so in analyzing the terms in (A3) on that boundary we must avoid the use of the inverse metric $q^{i j}$ as well as proper volume forms or unitnormalized normal vector/one-form fields. With these caveats in mind we note that:

$$
\tau^{a} g^{b c}\left(\nabla_{b} \delta g_{a c}-\nabla_{a} \delta g_{b c}\right)=\left(\tau^{a} \tilde{q}^{b c}+n^{a} \ell^{b} \mathcal{V}^{c}\right)\left(\nabla_{b} \delta g_{a c}-\nabla_{a} \delta g_{b c}\right)
$$

In turn, the two expressions on the right-hand side of this equations can be rewritten as:

$$
\begin{aligned}
& \tau^{a} \tilde{q}^{b c}\left(\nabla_{b} \delta g_{a c}-\nabla_{a} \delta g_{b c}\right) \\
& \quad=-2 \delta k_{\tau}-\delta \tilde{q}_{a b} k_{\tau}^{a b}+\tilde{q}^{a b} \nabla_{a} \delta \tau_{b}+\tilde{q}_{b}^{a} \nabla_{a} \delta \tau^{b}-\left(\delta \tilde{q}_{a b}\right) \tilde{q}^{a c}\left(\ell^{b} n^{d}+n^{b} \ell^{d}\right) \nabla_{c} \tau_{d},
\end{aligned}
$$


where $k_{\tau}=\tilde{q}^{a b} \nabla_{a} \tau_{b}$, and

$$
\begin{aligned}
& n^{a} \ell^{b} \mathcal{V}^{c}\left(\nabla_{b} \delta g_{a c}-\nabla_{a} \delta g_{b c}\right) \\
& \quad=-2 \delta \kappa_{\mathcal{V}}+2\left(\delta \mathcal{V}^{i}\right) \omega_{i}-\mathcal{V}^{b}\left(\delta n_{a} \nabla_{b} \ell^{a}+n_{a} \nabla_{b} \delta \ell^{a}+\delta n^{a} \nabla_{b} \ell_{a}+n^{a} \nabla_{b} \delta \ell_{a}\right) .
\end{aligned}
$$

There is still significant work needed to transform these expressions into what is present in (A1). We begin by considering the right hand side of equation (A6). First, we note that the $\delta k_{\tau}$ term can be rewritten using:

$$
\widetilde{\boldsymbol{\epsilon}} k_{\tau}=\widetilde{\boldsymbol{\epsilon}} \tilde{q}^{a b} \nabla_{a} \tau_{b}=-\tilde{\boldsymbol{\epsilon}} \tilde{q}^{a b} \nabla_{a} \mathcal{V}_{b}+2 \widetilde{\boldsymbol{\epsilon}} \alpha \theta_{(\ell)}=-\mathcal{L}_{\mathcal{V}} \widetilde{\boldsymbol{\epsilon}}+2 \widetilde{\boldsymbol{\epsilon}} \alpha \theta_{(\ell)}
$$

Then the $\mathcal{L}_{\mathcal{V}} \widetilde{\boldsymbol{\epsilon}}$ can be integrated out to the future and past boundaries of the horizon while the second term (and its variation) will vanish since we require that $\theta_{(\ell)}=0$ on the horizon. The second term in (A6) appears as a contribution to $\tilde{s}^{A B} \delta \tilde{q}_{A B}$ in the final expression ${ }^{12}$. Next, we turn to the third and fourth terms in equation (A6), those containing a $\delta \tau$. By expanding $\tau$ in terms of $\ell$ and $n$ using (3.5), it follows that they can be rewritten as

$$
\begin{aligned}
& \tilde{q}^{a b} \nabla_{a} \delta \tau_{b}+\tilde{q}_{b}^{a} \nabla_{a} \delta \tau^{b} \\
& \quad=2(\delta \alpha) \theta_{(\ell)}+2 \delta(\alpha C) \theta_{(n)}+\tilde{q}^{a b} \nabla_{a}\left(\tilde{q}_{b c} \delta \tau^{c}\right)-\left(\tilde{q}^{a b} \mathcal{L}_{\mathcal{V}} \tilde{q}_{a b}\right)\left(n^{a} \delta \ell_{a}+n_{a} \delta \ell^{a}\right) .
\end{aligned}
$$

The first two terms arising above appear in the final formula (A1) and the third is exact and will therefore vanish upon integration over a two surface $S_{v}$. The final term will combine with others to form an exact derivative. To see this, we add the last term on the right hand side of (A6) to the last term of (A7). By making use of the fact that $n^{a} \nabla_{b} \tau_{a}=n^{a} \nabla_{b} \mathcal{V}_{a}$ and $\ell^{a} \nabla_{b} \tau_{a}=-\ell^{a} \nabla_{b} \mathcal{V}_{a}$ it follows that

$$
\begin{aligned}
& -\left(\delta \tilde{q}_{a b}\right) \tilde{q}^{a c}\left(\ell^{b} n^{d}+n^{b} \ell^{d}\right) \nabla_{c} \tau_{d}-\mathcal{V}^{b}\left(\delta n_{a} \nabla_{b} \ell^{a}+n_{a} \nabla_{b} \delta \ell^{a}+\delta n^{a} \nabla_{b} \ell_{a}+n^{a} \nabla_{b} \delta \ell_{a}\right) \\
& \quad=-\mathcal{L}_{\mathcal{V}}\left(n^{a} \delta \ell_{a}+n_{a} \delta \ell^{a}\right) .
\end{aligned}
$$

When added to the $\mathcal{L}_{\mathcal{V}}$ term from (A9) we obtain an exact derivative which can be integrated out to the future and past horizon surfaces $S_{1}$ and $S_{2}$.

Finally, we turn our attention to the expressions in (A7). The first appears in the $\tilde{s}^{A B} \delta \tilde{q}_{A B}$ of the final expression (through $\tilde{q}^{A B} \delta \tilde{q}_{A B}=\tilde{q}^{a b} \delta \tilde{q}_{a b}$ ) while the last was dealt with in (A10). Therefore, we are left only with the $2\left(\delta \mathcal{V}^{i}\right) \omega_{i}$. This can be rewritten using $\delta\left(\mathcal{V}^{i} \mathbf{d} \boldsymbol{v}\right) \wedge \widetilde{\boldsymbol{\epsilon}}=$ $-p_{A}^{i}\left(\delta \tilde{V}^{A}\right) \mathbf{d} \boldsymbol{v} \wedge \widetilde{\boldsymbol{\epsilon}}$ to show that

$$
2 \mathbf{d} \boldsymbol{v} \wedge \tilde{\boldsymbol{\epsilon}}\left(\delta \mathcal{V}^{i}\right) \omega_{i}=-2 \delta\left(\mathbf{d} \boldsymbol{v} \wedge \tilde{\boldsymbol{\epsilon}} \tilde{V}^{A} \tilde{\omega}_{A}\right)-2(\delta \mathbf{d} \boldsymbol{v}) \wedge \tilde{\boldsymbol{\epsilon}} \kappa \mathcal{V}+2 \tilde{V}^{A} \delta\left(\mathbf{d} \boldsymbol{v} \wedge \tilde{\boldsymbol{\epsilon}} \tilde{\omega}_{A}\right)
$$

The results from (A8 - A11) can be used to rewrite the horizon terms from (A3) as:

$$
\begin{aligned}
& \int_{H} \mathbf{d} \boldsymbol{v} \wedge \tilde{\boldsymbol{\epsilon}} \tau^{a} g^{b c}\left(\nabla_{b}\left(\delta g_{a c}\right)-\nabla_{a}\left(\delta g_{b c}\right)\right)=-2 \delta\left(\int_{H}\left\{\mathbf{d} \boldsymbol{v} \wedge \tilde{\boldsymbol{\epsilon}}\left(\kappa_{\mathcal{V}}+\tilde{V}^{A} \tilde{\omega}_{A}\right)+2 \underline{\boldsymbol{n}} \theta_{(\ell)} \wedge \tilde{\boldsymbol{\epsilon}}\right\}\right) \\
& +\int_{H} \mathbf{d} \boldsymbol{v} \wedge \tilde{\boldsymbol{\epsilon}}\left[\left(\tilde{q}^{A B} k_{\tau}-k_{\tau}^{A B}+\tilde{q}^{A B} \kappa_{\mathcal{V}}\right) \delta \tilde{q}_{A B}\right] \\
& +\int_{H}\left\{2(\delta \underbrace{\ell}_{\leftarrow}) \wedge \tilde{\boldsymbol{\epsilon}} \theta_{(n)}-2(\delta \underline{\boldsymbol{n}}) \wedge \tilde{\boldsymbol{\epsilon}} \theta_{(\ell)}+\tilde{V}^{A} \delta\left(\mathbf{d} \boldsymbol{v} \wedge \tilde{\boldsymbol{\epsilon}} \tilde{\omega}_{A}\right)\right\} \\
& +\int_{S_{2}-S_{1}}\left\{2 \delta \widetilde{\boldsymbol{\epsilon}}-\widetilde{\boldsymbol{\epsilon}}\left[n_{b} \delta \ell^{b}+n^{b} \delta \ell_{b}\right]\right\}
\end{aligned}
$$

12 To be precise, note that $k_{\tau}^{a b} \delta \tilde{q}_{a b}=-k_{\tau a b} \delta \tilde{q}^{a b}=-k_{\tau a b} \delta\left(p_{A}^{a} p_{B}^{b} \tilde{q}^{A B}\right)=-k_{\tau A B} \delta \tilde{q}^{A B}=k_{\tau}^{A B} \delta q_{A B}$. 
In the above, $(\underline{\boldsymbol{\ell}})_{i}={p_{i}}^{a} \ell_{a}=\alpha C[d v]_{i}$ and $(\underline{\boldsymbol{n}})_{i}=p_{i}{ }^{a} n_{a}=\alpha[d v]_{i}$. By using the expression (3.11) for $\tilde{s}^{A B}$ we see that contribution on $H$ is of the form arising in (A1), leaving us only the corner terms arising at $S_{1}$ and $S_{2}$ to consider. We have a contribution $n_{b} \delta \ell^{b}+n^{b} \delta \ell_{b}$ arising from $H$ and another contribution, $\hat{n}_{a} \delta \hat{u}^{a}$ from $\Sigma_{1,2}$. However, there are relationships between the unit vectors $\hat{u}, \hat{n}$ and $\ell, n$ given in (3.2). Making use of these, and the fact that $\delta \hat{u}_{a} \propto \hat{u}_{a}$, it follows that

$$
\hat{n}^{a} \delta \hat{u}_{a}+n_{a} \delta \ell^{a}+n^{a} \delta \ell_{a}=-2 \delta(\ln \zeta) .
$$

Then, combining (A12), (A13) and recalling terms in the action (3.8) on $\Sigma_{1,2}$ and $H$, it is straightforward to arrive at the desired result, (A1).

\section{APPENDIX B: MANIPULATING THE BULK SYMPLECTIC STRUCTURE}

In this appendix, we will rewrite the bulk symplectic structure in a form which is directly useful in the body of the paper. We are interested in the case where $\delta_{1}=\delta_{\Lambda}$ generates an evolution along the vector field $\Lambda^{a}$. In three-dimensional language, the evolution of the three-metric, $h_{i j}$ and its conjugate momentum $P^{i j}$ which encodes the extrinsic curvature degrees of freedom are given in by:

$$
\delta_{\Lambda} h_{i j}=\lambda_{o} \frac{d}{d t} h_{i j}+\mathcal{L}_{\lambda} h_{i j} \quad \text { and } \quad \delta_{\Lambda} P^{i j}=\lambda_{o} \frac{d}{d t} P^{i j}+\mathcal{L}_{\lambda} P^{i j}
$$

If the bulk fields are required to satisfy the Einstein equations, then we can easily derive expressions for the time evolution of $h_{i j}$ and $P^{i j}$ as:

$$
\begin{aligned}
\frac{d}{d t} h_{i j}= & 64 \pi G N\left(P_{i j}-\frac{1}{2} P h_{i j}\right)+\mathcal{L}_{V} h_{i j} . \\
\frac{d}{d t} \mathbf{P}^{i j}= & \frac{{ }^{(\Sigma)} \boldsymbol{\epsilon}}{16 \pi G}\left(N\left(R^{i j}-\frac{1}{2} R h^{i j}\right)-\left(D^{i} D^{j} N-h^{i j} D_{k} D^{k} N\right)\right)+\mathcal{L}_{V} \mathbf{P}^{i j} \\
& +8 \pi G N\left(\left(P^{k l} \mathbf{P}_{k l}-\frac{1}{2} P \mathbf{P}\right) h^{i j}-4\left(P^{k(i} P_{k}^{j)}-\frac{1}{2} P \mathbf{P}^{i j}\right)\right),
\end{aligned}
$$

where $R$ is the three-dimensional Ricci scalar and $P=h_{i j} P^{i j}$ and $N$ and $V^{a}$ are the lapse and shift respectively. Then, a fairly standard calculation [14, 34] allows us to rewrite the bulk symplectic structure as:

$$
\begin{aligned}
& \int_{\Sigma}\left\{\left(\delta \mathbf{P}^{i j}\right)\left(\delta_{\Lambda} h_{i j}\right)-\left(\delta_{\Lambda} \mathbf{P}^{i j}\right)\left(\delta h_{i j}\right)\right\} \\
= & \int_{\Sigma}\left\{\lambda_{o} N \delta \boldsymbol{\mathcal { H }}+\left(\lambda^{i}+\lambda_{o} V^{i}\right) \delta \mathcal{H}_{i}\right\} \\
& +\int_{\partial \Sigma} \tilde{\boldsymbol{\epsilon}}\left\{-\lambda_{o}\left(\hat{n}_{k} V^{k}\right) \tilde{P}_{C D} \tilde{q}^{C A} \tilde{q}^{D B}+\frac{N \lambda_{o}}{16 \pi G}\left(k_{\hat{n}} \tilde{q}^{A B}-k_{\hat{n}}^{A B}\right)+\frac{\lambda_{o} \hat{n}^{i} D_{i} N}{16 \pi G} \tilde{q}^{A B}\right\} \delta \tilde{q}_{A B} \\
& +\int_{\partial \Sigma} 2 \delta\left(\widetilde{\boldsymbol{\epsilon}} \hat{n}^{i} P_{i j} p_{A}^{j}\right)\left(\lambda_{o} \tilde{V}^{A}+\tilde{\lambda}^{A}\right)+\int_{\partial \Sigma}\left\{2 \lambda_{o}\left(\hat{n}_{i} V^{i}\right) \delta\left(\widetilde{\boldsymbol{\epsilon}} P^{i j} \hat{n}_{i} \hat{n}_{j}\right)-\frac{N \lambda_{o}}{8 \pi G} \delta\left(\widetilde{\boldsymbol{\epsilon}} k_{\hat{n}}\right)\right\},
\end{aligned}
$$

where in the interests on conciseness we use tildes to represent two-dimensional quantities on all boundaries. Thus, $\tilde{P}_{A B}=p_{A}^{i} p_{B}^{j} P_{i j}$ is the pull-back of $P_{i j}, \tilde{V}^{A}$ and $\tilde{\lambda}^{A}$ are the components 
of $V^{i}$ and $\lambda^{i}$ in $T(\partial \Sigma)$, and $\tilde{q}_{A B}$ is the two-dimensional metric on $\partial \Sigma$. Additionally, since $\hat{n}$ is a unit vector in $\Sigma$ but orthogonal to $\partial \Sigma, k_{\hat{n}}^{A B}$ is the extrinsic curvature of the boundary $\partial \Sigma$ in $\Sigma$.

Now, we would like to rewrite the boundary contributions in terms of quantities which are tailored to the boundaries $B$ and $H$. In the four-dimensional context, we are more interested in the normals to $B$ and $H$ in $M$ rather than $\hat{n}$, the normal to $\partial \Sigma$ in $\Sigma$. The former are independent of our spacetime slicing while the latter depends upon the foliation of the spacetime. To facilitate this procedure, we re-express the familiar boundary quantities from Section III in terms of the three-dimensional quantites appearing above. We begin by writing out the various horizon quantities in terms of the three-dimensional metric and extrinsic curvature as:

$$
\begin{aligned}
\tilde{\omega}_{A}= & (16 \pi G) p_{A}{ }^{i} P_{i j} \hat{n}^{j}+d_{A}(\ln \zeta) \\
\kappa_{\mathcal{V}}= & \frac{d}{d t}(\ln \zeta)-\mathcal{L}_{\tilde{V}} \ln \zeta+16 \pi G\left(V^{k} n_{k}\right)\left[P_{i j} n^{i} n^{j}-P^{2} / 2\right]+\hat{n}^{i} D_{i} N \\
\theta_{(\ell)}= & \zeta\left[-(16 \pi G) P_{i j} \hat{n}^{i} \hat{n}^{j}+D_{i} \hat{n}^{i}\right] \\
\theta_{(n)}= & \frac{-1}{2 \zeta}\left[(16 \pi G) P_{i j} \hat{n}^{i} \hat{n}^{j}+D_{i} \hat{n}^{i}\right] \\
k_{\tau}^{A B}= & \left(16 \pi G \hat{n}_{i} V^{i}\right) \tilde{q}^{A C} \tilde{q}^{B D} \tilde{P}_{C D}+N k_{\hat{n}}^{A B} \\
\tilde{s}^{A B}= & -\left(16 \pi G V^{i} \hat{n}_{i}\right) \tilde{q}^{A C} \tilde{q}^{B D} \tilde{P}_{C D}+N\left(k_{\hat{n}} \tilde{q}^{A B}-k_{\hat{n}}^{A B}\right)+\left(\hat{n}^{i} D_{i} N\right) \tilde{q}^{A B} \\
& +\tilde{q}^{A B}\left[\frac{d}{d t}(\ln \zeta)-\mathcal{L}_{\tilde{V}}(\ln \zeta)\right]
\end{aligned}
$$

Similarly, we can write the outer boundary terms as:

$$
\begin{aligned}
k_{\bar{n}}^{A B}= & \sqrt{1+\eta^{2}} k_{\hat{n}}^{A B}+(16 \pi G \eta)\left[\bar{\gamma}^{A C} \bar{\gamma}^{B D} \tilde{P}_{C D}-P \bar{\gamma}^{A B} / 2\right] \\
\bar{n}^{c} \Delta_{c} \bar{N}= & \hat{n}^{i} D_{i} N+16 \pi G\left(V^{k} \hat{n}_{k}\right)\left[\hat{n}^{i} \hat{n}^{j} P_{i j}-P / 2\right]+\frac{d}{d t}\left(\sinh ^{-1} \eta\right)-\mathcal{L}_{\bar{V}}\left(\sinh ^{-1} \eta\right)( \\
\bar{\gamma}_{A}= & -16 \pi G p_{A}^{i} \tilde{P}_{i j} \hat{n}^{j}-d_{A}\left(\sinh ^{-1} \eta\right) \\
\bar{N} \bar{s}^{A B}= & -\left(16 \pi G \hat{n}_{i} V^{i}\right) \bar{\gamma}^{A C} \bar{\gamma}^{B D} \tilde{P}_{C D}+N\left(k_{\hat{n}} \bar{\gamma}^{A B}-k_{\hat{n}}^{A B}\right)+\left(\hat{n}^{i} D_{i} N\right) \bar{\gamma}^{A B} \\
& +\bar{\gamma}^{A B}\left[\frac{d}{d t}\left(\sinh ^{-1} \eta\right)-\mathcal{L}_{\bar{V}}\left(\sinh ^{-1} \eta\right)\right]
\end{aligned}
$$

With these expressions at hand, it is a reasonably straightforward exercise to substitute 
them into (B4). We thereby obtain:

$$
\begin{aligned}
& \int_{\Sigma}\left\{\left(\delta \mathbf{P}^{i j}\right)\left(\delta_{\Lambda} h_{i j}\right)-\left(\delta_{\Lambda} \mathbf{P}^{i j}\right)\left(\delta h_{i j}\right)\right\} \\
= & \delta\left(H_{\Lambda}-\int_{\mathcal{B}} \overline{\boldsymbol{\epsilon}}\left\{\frac{\lambda_{o}}{8 \pi G} \bar{N} k_{\bar{n}}+\left(\lambda_{o} \bar{V}^{A}+\lambda^{A}\right) \bar{j}_{A}\right\}+\frac{1}{8 \pi G} \int_{S} \widetilde{\boldsymbol{\epsilon}} \tilde{\lambda}^{A} \tilde{\omega}_{A}\right) \\
& -\int_{\Sigma}\left\{\delta\left(\lambda_{o} N\right) \mathcal{H}+\delta\left(\lambda^{i}+\lambda_{o} V^{i}\right) \mathcal{H}_{i}\right\} \\
& \left.+\frac{1}{8 \pi G} \int_{S}\left\{\left(\delta_{\Lambda} \widetilde{\boldsymbol{\epsilon}}\right)(\delta \ln \zeta)-(\delta \widetilde{\boldsymbol{\epsilon}})\left(\delta_{\Lambda} \ln \zeta\right)\right\}-\frac{1}{8 \pi G} \int_{S}(\delta \tilde{\lambda})^{A}\right) \tilde{\boldsymbol{\epsilon}}^{\tilde{\omega}_{A}} \\
& +\frac{\lambda_{o}}{8 \pi G} \int_{S}\left\{\frac{1}{2} \widetilde{\boldsymbol{\epsilon}} \tilde{s}^{A B} \delta \tilde{q}_{A B}+\tilde{V}^{A} \delta\left(\tilde{\omega}_{A} \widetilde{\boldsymbol{\epsilon}}\right)+\delta(\alpha C) \theta_{(n)} \widetilde{\boldsymbol{\epsilon}}+(\delta \alpha) \widetilde{\boldsymbol{\epsilon}} \theta_{(\ell)}+2 \delta\left(\alpha \widetilde{\boldsymbol{\epsilon}} \theta_{(\ell)}\right)-\delta\left(\frac{d \widetilde{\boldsymbol{\epsilon}}}{d t}\right)\right\} \\
& +\frac{1}{8 \pi G} \int_{\mathcal{B}}\left\{\left(\delta_{\Lambda} \sinh ^{-1} \eta\right)(\delta \overline{\boldsymbol{\epsilon}})-\delta\left(\sinh ^{-1} \eta\right)\left(\delta_{\Lambda} \overline{\boldsymbol{\epsilon}}\right)\right\} \\
& +\int_{\mathcal{B}} \overline{\boldsymbol{\epsilon}}\left\{\frac{\lambda_{o} \bar{N}}{16 \pi G} \bar{s}^{A B} \delta \bar{\gamma}_{A B}+\bar{\jmath}_{A} \delta\left(\lambda_{o} \bar{V}^{A}+\bar{\lambda}^{A}\right)+\frac{\delta\left(\bar{N} \lambda_{o}\right)}{8 \pi G} k_{\bar{n}}\right\} .
\end{aligned}
$$

In the above, we have introduced the notation $H_{\Lambda}$ to signify the bulk part of the Hamiltonian, namely

$$
H_{\Lambda}=\int_{\Sigma} d^{3} x\left\{\lambda_{o}\left(N \mathcal{H}+V^{i} \mathcal{H}_{i}\right)+\lambda^{i} \mathcal{H}_{i}\right\}
$$

We can now interpret the terms arising in the expression (B14) one at a time. The first term is an exact variation, which is precisely what is required. This will be part of the Hamiltonian which generates the evolution along $\Lambda$. The next term consists of variations of the lapse and shift multiplying the bulk constraints. This will clearly vanish provided the bulk constraints are satisfied. The remaining terms are all boundary terms. On each boundary, we have a contribution containing terms involving the variation of the boundary volume forms $\widetilde{\boldsymbol{\epsilon}}$ and $\overline{\boldsymbol{\epsilon}}$. From these, we identify the momenta conjugate to the volume forms as:

$$
\tilde{P}=\frac{1}{8 \pi G} \ln \zeta \quad \text { and } \quad \bar{P}=\frac{1}{8 \pi G} \sinh ^{-1} \eta
$$

The equations of motion then guarantee that the evolution of these quantities along $\Lambda$ is given by $\mathcal{L}_{\Lambda}$ as expected.

Finally we come to the remaining boundary terms at the horizon and outer boundary. In all cases, these terms either vanish (because $\theta_{(\ell)}=0$ ) or the quantity which appears inside variation is held "fixed" by our boundary conditions. Although these quantities are held fixed, with the weak boundary conditions introduced in Section IIID, their values will change under diffeomorphisms of the boundary. Thus, variations of these quantities do not vanish, but are equal to Lie derivatives along a direction $X^{i}$ tangent to the boundary surface. For a three-dimensional Hamiltonian formulation this means that

$$
\delta_{X}=\delta t\left(\frac{\partial}{\partial t}\right)+\mathcal{L}_{\widetilde{\delta X}},
$$

as discussed in (4.5). 
In addition, we require that the diffeomorphism constraint be satisfied at both the horizon and outer boundary. The analysis of the outer boundary was performed in detail in [15]. We refer the interested reader to that paper (and in particular Appendix C); here we will simply use the result to rewrite the terms arising in $(\mathrm{B} 14)$. Therefore, we turn our attention to the horizon. On $S$, the diffeomorphism constraint can be rewritten in a form (3.20) which is directly applicable for our current calculation (B14). The constraint equation on the horizon allows us to immediately cancel all the terms other than the $\tilde{\omega}_{A}$ ones. These can be rewritten using:

$$
\int_{S} \tilde{x}^{A} \mathcal{L}_{\mathcal{V}}\left(\widetilde{\boldsymbol{\epsilon}} \tilde{\omega}_{A}\right)=\int_{S}\left\{\widetilde{\delta X}^{A} \frac{d}{d t}\left(\widetilde{\boldsymbol{\epsilon}} \tilde{\omega}_{A}\right)+\tilde{V}^{A} \delta_{X}\left(\widetilde{\boldsymbol{\epsilon}} \tilde{\omega}_{A}\right)\right\}
$$

Additionally, we must express the $\delta \tilde{\lambda}^{A}$ term in our preferred co-ordinate system. As in [15], we can make use of the relation

$$
\delta \tilde{\lambda}^{\alpha}=(\delta \tilde{\lambda})^{A}\left(d_{A} \tilde{\theta}^{\alpha}\right)-\tilde{\lambda}^{A} d_{A}\left(\delta \tilde{\theta}^{\alpha}\right)
$$

to rewrite

$$
\int_{S}\left(\delta \tilde{\lambda}^{A}\right) \widetilde{\boldsymbol{\epsilon}} \tilde{\omega}_{A}+\lambda_{o}\left(\delta \tilde{\theta}^{A}\right) \mathcal{L}_{T}\left(\widetilde{\boldsymbol{\epsilon}} \tilde{\omega}_{A}\right)=\int_{S}\left(\delta \tilde{\lambda}^{\alpha}\right) \widetilde{\boldsymbol{\epsilon}} \tilde{\omega}_{\alpha}+\delta \tilde{\theta}^{\alpha} \mathcal{L}_{\Lambda}\left(\widetilde{\boldsymbol{\epsilon}} \tilde{\omega}_{\alpha}\right)
$$

Finally combining everything from above, we can write the bulk symplectic structure as:

$$
\begin{aligned}
& \int_{\Sigma}\left\{\left(\delta \mathbf{P}^{i j}\right)\left(\delta_{\Lambda} h_{i j}\right)-\left(\delta_{\Lambda} \mathbf{P}^{i j}\right)\left(\delta h_{i j}\right)\right\} \\
& =\delta\left(H_{\Lambda}-\int_{\mathcal{B}}\left\{\frac{\lambda_{o}}{8 \pi G} \overline{\boldsymbol{\epsilon}} \bar{N} k_{\bar{n}}+\overline{\boldsymbol{\epsilon}}\left(\lambda_{o} \bar{V}^{A}+\lambda^{A}\right) \bar{\jmath}_{A}\right\}+\frac{1}{8 \pi G} \int_{S} \tilde{\boldsymbol{\epsilon}} \tilde{\lambda}^{A} \tilde{\omega}_{A}\right) \\
& -\int_{\Sigma}\left\{\delta\left(\lambda_{o} N\right) \mathcal{H}+\delta\left(\lambda^{i}+\lambda_{o} V^{i}\right) \mathcal{H}_{i}\right\} \\
& +\int_{\mathcal{B}}\left\{\left(\delta_{\Lambda} \bar{P}\right)(\delta \overline{\boldsymbol{\epsilon}})-(\delta \bar{P})\left(\delta_{\Lambda} \overline{\boldsymbol{\epsilon}}\right)\right\}+\int_{S}\left\{\left(\delta_{\Lambda} \tilde{\boldsymbol{\epsilon}}\right)(\delta \tilde{P})-(\delta \widetilde{\boldsymbol{\epsilon}})\left(\delta_{\Lambda} \tilde{P}\right)\right\} \\
& +\left(\delta \lambda_{o}\right) \int_{\mathcal{B}}\left\{\frac{\bar{N}}{8 \pi G} \overline{\boldsymbol{\epsilon}} k_{\bar{n}}+\overline{\boldsymbol{\epsilon}} \bar{\jmath}_{A} \bar{V}^{A}\right\}+\delta t \int_{\mathcal{B}} \mathcal{L}_{\Lambda}\left\{\frac{\bar{N}}{8 \pi G} \overline{\boldsymbol{\epsilon}} k_{\bar{n}}+\overline{\boldsymbol{\epsilon}} \bar{\jmath}_{A} \bar{V}^{A}\right\} \\
& +\int_{\mathcal{B}}\left\{\left(\delta \bar{\lambda}^{\alpha}\right) \overline{\boldsymbol{\epsilon}} \bar{\jmath}_{\alpha}+\delta \bar{\theta}^{\alpha} \delta_{\Lambda}\left(\overline{\boldsymbol{\epsilon}} \bar{\jmath}_{\alpha}\right)\right\}-\frac{1}{8 \pi G} \int_{S}\left\{\left(\delta \tilde{\lambda}^{\alpha}\right) \widetilde{\boldsymbol{\epsilon}} \tilde{\omega}_{\alpha}+\delta \tilde{\theta}^{\alpha} \delta_{\Lambda}\left(\widetilde{\boldsymbol{\epsilon}} \tilde{\omega}_{\alpha}\right)\right\} .
\end{aligned}
$$

This is the final desired form.

[1] S.W. Hawking and G.F.R. Ellis, The large scale structure of space-time, (Cambridge University Press, 1973).

[2] J. M. Bardeen, B. Carter and S. W. Hawking, Commun. Math. Phys. 31, 161 (1973).

[3] J. D. Bekenstein, Phys. Rev. D 7, 2333 (1973).

[4] S. W. Hawking, Commun. Math. Phys. 43, 199 (1975).

[5] R. Penrose, Phys. Rev. Lett. 14, 57 (1965).

[6] S.A. Hayward, Phys.Rev. D49 6467 (1994).

[7] S.A. Hayward, Phys.Rev. D70 104027 (2004). S.A. Hayward, Phys.Rev.Lett. 93251101 (2004). 
[8] A. Ashtekar, C. Beetle and J. Lewandowski, Phys.Rev. D64 044016 (2001); A. Ashtekar et. al., Phys.Rev.Lett 853564 (2000); A. Ashtekar, S. Fairhurst and B. Krishnan, Phys.Rev. D62 104025 (2000); A. Ashtekar, C. Beetle and S. Fairhurst, Class.Quantum Grav. 17253 (2000).

[9] A. Ashtekar and B. Krishnan, Phys.Rev.Lett. 89261101 (2002); Phys.Rev. D68 104030 (2003).

[10] I. Booth and S. Fairhurst, Phys.Rev.Lett 92011102 (2004).

[11] J.D. Brown and J.W. York, Phys. Rev. D47 1407 (1993).

[12] J.D. Brown and J.W. York, Phys. Rev. D47 1421 (1993).

[13] I.S. Booth and R.B. Mann, Phys. Rev. D59, 064021 (1999).

[14] J.D. Brown, S.R. Lau and J.W. York, Ann. Phys. NY 297, 175 (2002).

[15] I. Booth and S. Fairhurst, Class. Quantum Grav. 20, 4507 (2003).

[16] R.M. Wald, General Relativity (University of Chicago Press, 1984).

[17] W. Israel, Can. J. Phys. 64 120-127 (1986); Phys. Rev. Lett. 56, 789-791 (1986).

[18] M. Kriele and S. Hayward, J. Math. Phys. 381593 (1997).

[19] P.T. Chruściel, Lect.Notes Phys. 604 61-102 (2002).

[20] T.W. Baumgarte and S.L. Shapiro, Physics Reports 37641 (2003).

[21] R.M. Wald and V. Iyer, Phys.Rev. D44 3719 (1991).

[22] A. Ashtekar, J. C. Baez and K. Krasnov, Adv. Theor. Math. Phys. 4, 1 (2000); A. Ashtekar, J. Engle and C. Van Den Broeck, Class. Quantum Grav. 22 L27 (2005).

[23] I. Ben-Dov, Phys.Rev. D70 124031 (2004).

[24] I. Booth, L. Brits, J. Gonzalez and C. Van Den Broeck, "Marginally Trapped Tubes and Dynamical Horizons", gr-qc/0506119 (2005).

[25] A. Ashtekar and G. Galloway, "Some Uniqueness Results for Dynamical Horizons", gr-qc/0503109 (2005).

[26] E. Poisson, A Relativist's Toolkit: The Mathematics of Black-Hole Mechanics (Cambridge University Press, 2004).

[27] R.J. Epp, Phys.Rev. D62, 124018 (2000).

[28] G. Hayward, Phys.Rev. D47, 3275 (1993).

[29] I. Booth and S. Fairhurst, "Perturbatively non-isolated trapping horizons", In Preparation.

[30] S.R. Lau, Phys.Rev D60, 104034 (1999); P. Krauss, F. Larsen, and R. Sieblink, Nucl.Phys. B563, 259 (1999); V. Balasubramnanian and P. Krauss, Commun. Math. Phys. 208, 413 (1999); R.B. Mann, Phys.Rev D60, 104047 (1999); Phys.Rev. D61, 084010 (2000).

[31] R. Arnowitt, S. Deser, and C.W. Misner, The dynamics of general relativity. In Gravitation: An introduction to the current research, L.Witten, editor, (New York, Wiley) (1962).

[32] V.I. Arnold, Mathematical Methods of Classical Mechanics (Springer-Verlag, New York, 1989).

[33] J. Kijowski, Gen. Rel. Grav. 29, 307 (1997); K. Kuchař Phys. Rev. D50, 3961 (1994).

[34] I.S. Booth, A quasi-local Hamiltonian for gravity with classical and quantum applications, (PhD Thesis, University of Waterloo, 2000), gr-qc/0008030.

[35] L. Andersson, M. Mars and W. Simon, "Local existence of dynamical and trapping horizons", Phys. Rev. Lett. 95111102 (2005). 